\title{
Review Article \\ Color Confinement, Hadron Dynamics, and Hadron Spectroscopy from Light-Front Holography and Superconformal Algebra
}

\author{
Stanley J. Brodsky \\ SLAC National Accelerator Laboratory, Stanford University, Stanford, CA, USA \\ Correspondence should be addressed to Stanley J. Brodsky; sjbth@slac.stanford.edu
}

Received 12 June 2017; Accepted 31 January 2018; Published 16 April 2018

Academic Editor: Ralf Hofmann

Copyright (C) 2018 Stanley J. Brodsky. This is an open access article distributed under the Creative Commons Attribution License, which permits unrestricted use, distribution, and reproduction in any medium, provided the original work is properly cited. The publication of this article was funded by $\mathrm{SCOAP}^{3}$.

The QCD light-front Hamiltonian equation $H_{\mathrm{LF}}|\Psi\rangle=M^{2}|\Psi\rangle$ derived from quantization at fixed LF time $\tau=t+z / c$ provides a causal, frame-independent method for computing hadron spectroscopy as well as dynamical observables such as structure functions, transverse momentum distributions, and distribution amplitudes. The QCD Lagrangian with zero quark mass has no explicit mass scale. de Alfaro, Fubini, and Furlan (dAFF) have made an important observation that a mass scale can appear in the equations of motion without affecting the conformal invariance of the action if one adds a term to the Hamiltonian proportional to the dilatation operator or the special conformal operator. If one applies the dAFF procedure to the QCD light-front Hamiltonian, it leads to a color-confining potential $\kappa^{4} \zeta^{2}$ for mesons, where $\zeta^{2}$ is the LF radial variable conjugate to the $q \bar{q}$ invariant mass squared. The same result, including spin terms, is obtained using light-front holography, the duality between light-front dynamics and $\mathrm{AdS}_{5}$, if one modifies the $\mathrm{AdS}_{5}$ action by the dilaton $e^{\kappa^{2} z^{2}}$ in the fifth dimension $z$. When one generalizes this procedure using superconformal algebra, the resulting light-front eigensolutions provide a unified Regge spectroscopy of meson, baryon, and tetraquarks, including remarkable supersymmetric relations between the masses of mesons and baryons and a universal Regge slope. The pion $q \bar{q}$ eigenstate has zero mass at $m_{q}=0$. The superconformal relations also can be extended to heavy-light quark mesons and baryons. This approach also leads to insights into the physics underlying hadronization at the amplitude level. I will also discuss the remarkable features of the Poincaré invariant, causal vacuum defined by light-front quantization and its impact on the interpretation of the cosmological constant. AdS/QCD also predicts the analytic form of the nonperturbative running coupling $\alpha_{s}\left(Q^{2}\right) \propto e^{-Q^{2} / 4 \kappa^{2}}$. The mass scale $\kappa$ underlying hadron masses can be connected to the parameter $\Lambda_{\overline{\mathrm{MS}}}$ in the QCD running coupling by matching the nonperturbative dynamics to the perturbative QCD regime. The result is an effective coupling $\alpha_{s}\left(Q^{2}\right)$ defined at all momenta. One obtains empirically viable predictions for spacelike and timelike hadronic form factors, structure functions, distribution amplitudes, and transverse momentum distributions. Finally, I address the interesting question of whether the momentum sum rule is valid for nuclear structure functions.

\section{Introduction}

A profound question in hadron physics is how the proton mass and other hadronic mass scales can be determined by QCD since there is no explicit parameter with mass dimensions in the QCD Lagrangian for vanishing quark mass. This dilemma is compounded by the fact that the chiral QCD Lagrangian has no knowledge of the conventions used for units of mass such as MeV. Thus QCD with $m_{q}=0$ can in principle only predict ratios of masses such as $m_{\rho} / m_{p}$, not their absolute values. Similarly, given that color is confined, how does QCD set its range without a parameter with dimensions of length? It is hard to see how this mass scale problem could be solved by "dimensional transmutation," since the mass scale determined by perturbative QCD such as $\Lambda_{\overline{\mathrm{MS}}}$ is renormalization scheme dependent, whereas hadron masses are independent of the conventions chosen to regulate the UV divergences. 
A remarkable principle, first demonstrated by de Alfaro, Fubini, and Furlan (dAFF) [8] for conformal theory in $1+1$ quantum mechanics, is that a mass scale can appear in a Hamiltonian and its equations of motion without affecting the conformal invariance of the action. The essential step introduced by dAFF is to add to the conformal Hamiltonian terms proportional to the dilation operator $D$ and the special conformal operator $K$. The unique result is the addition of a harmonic oscillator potential $V(x)=\kappa^{4} x^{2}$ to the Hamiltonian, The group algebra is maintained despite the fact that $D$ and $K$ have dimensions. In fact, the new Hamiltonian has "extended dilatation invariance" since the mass scale $\kappa$ can be rescaled arbitrarily. This implies that only ratios of the mass eigenvalues can be determined, not their absolute values.

Brodsky et al. [9] have shown that a mass gap and color confinement appears when one extends the dAFF procedure to relativistic, causal, Poincaré invariant, light-front Hamiltonian theory for QCD. The resulting predictions for both hadronic spectroscopy and dynamics provide an elegant description of meson and baryon phenomenology, including Regge trajectories with universal slopes in the principal quantum number $n$ and the orbital angular momentum $L$. In addition, the resulting quark-antiquark bound-state equation predicts a massless pion for zero quark mass.

In this contribution, I will review a number of recent advances in holographic QCD, extending earlier reviews given in [10-12] with a new emphasis on the impact of superconformal algebra and new applications. As I will discuss, the resulting light-front eigensolutions provide a unified Regge spectroscopy of meson, baryon, and tetraquarks, including remarkable supersymmetric relations between the masses of mesons and baryons and a universal Regge slope. The combination of light-front holography with superconformal algebra thus leads to the novel prediction that hadron physics has supersymmetric properties in both spectroscopy and dynamics. It also predicts the form of the QCD running coupling at all scales and provides new insights into the physics underlying hadronization at the amplitude level. I will also discuss the remarkable features of the Poincaré invariant, causal vacuum defined by light-front quantization and its impact on the interpretation of the cosmological constant. Finally, I address the interesting question of whether the momentum sum rule is valid for nuclear structure functions.

Light-Front quantization is the natural formalism for relativistic quantum field theory. Measurements of hadron structure, such as deep inelastic lepton-proton scattering, are made at fixed light-front time $\tau=t+z / c$, analogous to a flash photograph, not at a single "instant time." As shown by Dirac [13], boosts are kinematical in the "front form." Thus all formulae using the front form are independent of the observer's motion [14]; that is, they are Poincaré invariant. The eigenstates of the light-front Hamiltonian $H_{\mathrm{LF}}=P^{+} P^{-}-$ $\vec{P}_{\perp}^{2}$ derived from the QCD Lagrangian encode the entire the hadronic mass spectrum for both individual hadrons and the multihadron continuum. The eigenvalues of the LF Hamiltonian are the squares of the hadron masses $M_{H}^{2}$ : $H_{\mathrm{LF}}\left|\Psi_{H}\right\rangle=M_{H}^{2}\left|\Psi_{H}\right\rangle$ [14]. The evaluation of the Wilson line for gauge theories in the front form is discussed in [15]. In addition, I will discuss the advantages of perturbative QCD calculations using light-front-time-ordered perturbation theory, including the use of $J^{z}$ conservation.

The eigenfunctions of the light-front Hamiltonian $H_{\mathrm{LF}}=$ $P^{+} P^{-}-\vec{P}_{\perp}^{2}$ derived from the QCD Lagrangian correspond to the single hadron and multihadronic continuum eigenstates. The eigenvalues of the LF Hamiltonian are the squares of the hadron masses $M_{H}^{2}: H_{\mathrm{LF}}\left|\Psi_{H}\right\rangle=M_{H}^{2}\left|\Psi_{H}\right\rangle$ [14]. Here $P^{-}=i(d / d \tau)$ is the LF time evolution operator, and $P^{+}=$ $P^{0}+P^{z}$ and $\vec{P}_{\perp}$ are kinematical. The eigenfunctions of $H_{\mathrm{LF}}$ provide hadronic LF Fock state wavefunctions (LFWFs): $\psi_{n}^{H}\left(x_{i}, \vec{k}_{\perp i}, \lambda_{i}\right)=\left\langle n \mid \Psi_{H}\right\rangle$, the projection of the hadronic eigenstate on the free Fock basis. The constituents' physical momenta are $p_{i}^{+}=x_{i} P^{+}$and $\vec{p}_{\perp i}=x_{i} \vec{P}_{\perp}+\vec{k}_{\perp i}$, and the $\lambda_{i}$ label the spin projections $S_{i}^{z}$. Remarkably one can reduce the LF Hamiltonian theory for $q \bar{q}$ mesons with $m_{q}=0$ to an effective LF Schrödinger equation in a single variable, the LF radial variable $\zeta^{2}=b_{\perp}^{2} x(1-x)$.

The LFWFs are Poincaré invariant: they are independent of $P^{+}$and $P_{\perp}$ and are thus independent of the motion of the observer. Since the LFWFs are independent of the hadron's momentum, there is no length contraction $[16,17]$. Structure functions are essentially the absolute square of the LFWFs. One thus measures the same structure function in an electron-ion collider as in an electron-scattering experiment where the target hadron is at rest.

Light-front wavefunctions thus provide a direct link between the QCD Lagrangian and hadron structure. Since they are defined at a fixed $\tau$, they connect the physical on-shell hadronic state to its quark and gluon parton constituents, not at off-shell energy, but off-shell in invariant mass squared $\mathscr{M}^{2}=\left(\sum_{i} k_{i}^{\mu}\right)^{2}$. They thus control the transformation of the quarks and gluons in an off-shell intermediate state into the observed final on-shell hadronic state. See Figure 1.

One of the most elegant features of quantum field theory is supersymmetry, where fermionic and bosonic eigensolutions have the same mass. The conformal group has an elegant $2 \times 2$ Pauli matrix representation called superconformal algebra, originally discovered by Haag et al. [18] (1975). The conformal Hamiltonian operator and the special conformal operators can be represented as anticommutators of Pauli matrices $H=1 / 2\left[Q, Q^{\dagger}\right]$ and $K=1 / 2\left[S, S^{\dagger}\right]$. As shown by Fubini and Rabinovici [19], a nonconformal Hamiltonian with a mass scale and universal confinement can then be obtained by shifting $Q \rightarrow Q+\omega K$, the analog of the dAFF procedure. Thus the conformal algebra can be extended even though $\omega$ has dimension of mass. In effect one has generalized supercharges of the superconformal algebra [19]. The result of this shift of the Hamiltonian is a color-confining harmonic potential in the equations of motion. Remarkably the action remains conformally invariant, and only one mass parameter appears.

As shown by Guy de Téramond, Günter Dosch and myself, the bound-state equations of superconformal algebra are, in fact, Lorentz invariant, frame-independent, relativistic light-front Schrödinger equations, and the resulting eigensolutions are the eigenstates of a light-front Hamiltonian 


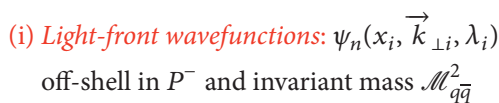

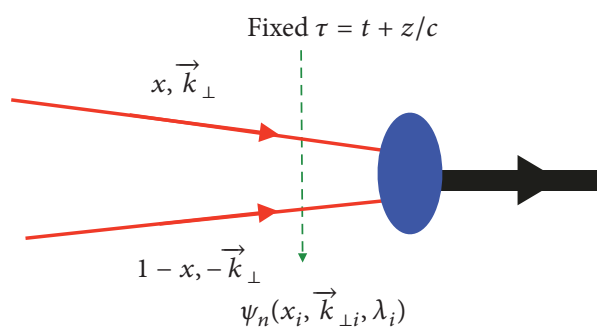

"Hadronization at the amplitude level"

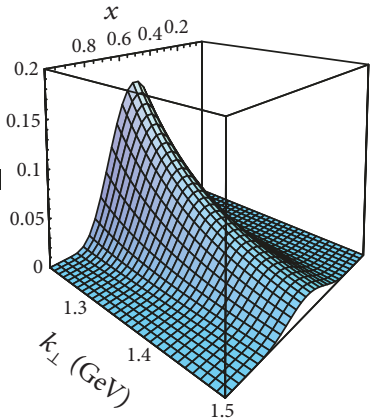

Boost-invariant LFWF connects confined quarks and gluons to hadrons

FIGURE 1: The meson LFWF connects the intermediate $q \bar{q}$ state, which is off of the $P^{-}$energy shell and thus off-the-invariant mass shell $\mathscr{M}^{2}>m_{H}^{2}$ to the physical meson state with $\mathscr{M}^{2}=m_{H}^{2} \cdot q$ and $\bar{q}$ can be regarded as effective dressed fields.

\section{LF holography Baryon equation}

$$
\begin{gathered}
\left(-\partial_{\zeta}^{2}+\kappa^{4} \zeta^{2}+2 \kappa^{2}\left(L_{B}+1\right)+\frac{4 L_{B}^{2}-1}{4 \zeta^{2}}\right) \psi_{J}^{+}=M^{2} \psi_{J}^{+} \quad \mathrm{G}_{22} \\
\left(-\partial_{\zeta}^{2}+\kappa^{4} \zeta^{2}+2 \kappa^{2} L_{B}+\frac{4\left(L_{B}+1\right)^{2}-1}{4 \zeta^{2}}\right) \psi_{J}^{-}=M^{2} \psi_{J}^{-} \quad \mathrm{G}_{11} \\
\begin{array}{cc}
M^{2}\left(n, L_{B}\right)=4 \kappa^{2}\left(n+L_{B}+1\right) & \mathrm{S}=1 / 2, \mathrm{P}=+ \\
\text { Meson equation } & \text { both chiralities }
\end{array}
\end{gathered}
$$

$$
\left(-\partial_{\zeta}^{2}+\kappa^{4} \zeta^{2}+2 \kappa^{2}(J-1)+\frac{4 L_{M}^{2}-1}{4 \zeta^{2}}\right) \phi_{J}=M^{2} \phi_{J} \quad \mathrm{G}_{11}
$$

$$
M^{2}\left(n, L_{M}\right)=4 \kappa^{2}\left(n+L_{M}\right) \quad \text { Same } \kappa !
$$

$S=0, I=1$ Meson is superpartner of $S=1 / 2, I=1$ baryon Meson-baryon degeneracy for $L_{M}=L_{B}+1$

FIGURE 2: The LF Schrödinger equations for baryons and mesons for zero quark mass derived from the Pauli $2 \times 2$ matrix representation of superconformal algebra. $\psi^{ \pm}$are the baryon quark-diquark LFWFs where the quark spin $S_{q}^{z}= \pm 1 / 2$ is parallel or antiparallel to the baryon spin $J^{z}= \pm 1 / 2$. The meson and baryon equations are identical if one identifies a meson with internal orbital angular momentum $L_{M}$ with its superpartner baryon with $L_{B}=L_{M}-1$. See [1-3].

obtained from $\mathrm{AdS}_{5}$ and light-front holography. Light-front quantization at fixed light-front time $\tau=t+z / c$ provides a physical, frame-independent formalism for hadron dynamics and structure.

Superconformal algebra leads to effective QCD lightfront bound-state equations for both mesons and baryons [13 ]. The resulting set of bound-state equations for confined quarks are shown in Figure 2. The supercharges connect the baryon and meson spectra and their Regge trajectories to

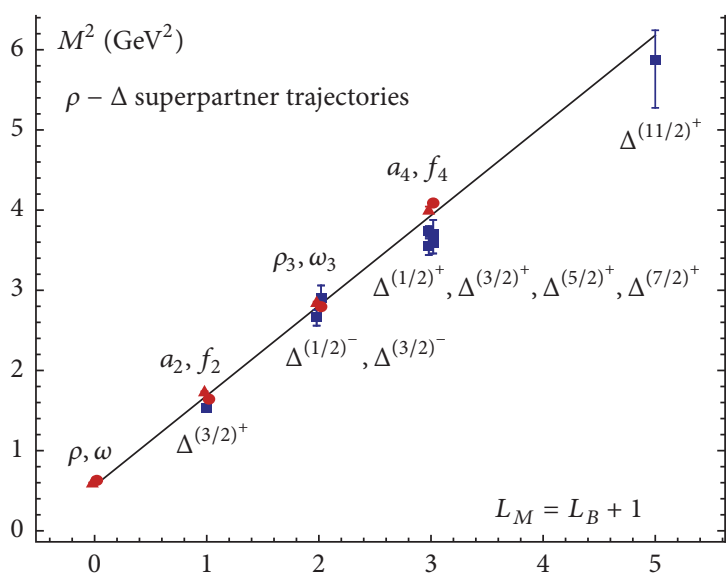

FIgURE 3: Comparison of the $\rho / \omega$ meson Regge trajectory with the $J=3 / 2 \Delta$ baryon trajectory. Superconformal algebra predicts the degeneracy of the meson and baryon trajectories if one identifies a meson with internal orbital angular momentum $L_{M}$ with its superpartner baryon with $L_{M}=L_{B}+1$. See $[1,2]$.

each other in a remarkable manner: the superconformal algebra predicts that the bosonic meson and fermionic baryon masses are equal if one identifies each meson with internal orbital angular momentum $L_{M}$ with its superpartner baryon with $L_{B}=L_{M}-1$; the meson and baryon superpartners then have the same parity. Since $2+L_{M}=3+L_{B}$, the twist-dimension of the meson and baryon superpartners are also the same. Superconformal algebra thus explains the phenomenological observation that Regge trajectories for both mesons and baryons have parallel slopes.

The comparison between the meson and baryon masses of the $\rho / \omega$ Regge trajectory with the spin-3/2 $\Delta$ trajectory is shown in Figure 3. The observed hadronic spectrum with $N_{C}=3$ is seen to exhibit the supersymmetric features predicted by superconformal algebra. 


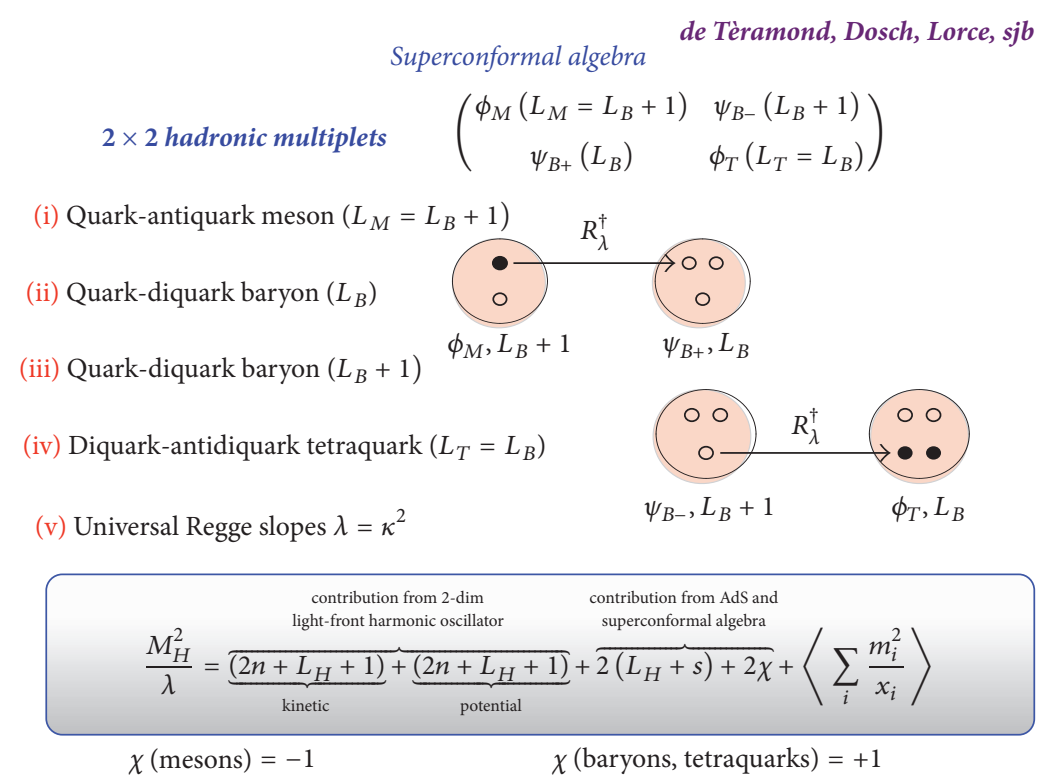

FIGURE 4: The eigenstates of superconformal algebra have a $2 \times 2$ representation of mass degenerate bosons and fermions: a meson with $L_{M}=L_{B}+1$, a baryon doublet with $L_{B}, L_{B}+1$ components, and a tetraquark with $L_{T}=L_{B}$. The breakdown of LF kinetic, potential, spin, and quark mass contributions to each hadron is also shown. The virial theorem predicts the equality of the LF kinetic and potential contributions.

As illustrated in Figure 4, the hadronic eigensolutions of the superconformal algebra are $2 \times 2$ matrices connected internally by the supersymmetric algebra operators. The eigensolutions of the supersymmetric conformal algebra thus have a $2 \times 2$ Pauli matrix representation, where the upper-left component corresponds to mesonic $q \bar{q}$ colorsinglet bound states; the two off-diagonal eigensolutions $\psi^{ \pm}$correspond to a pair of Fock components of baryonic quark-diquark states with equal weight, where the quark spin is parallel or antiparallel to the baryon spin, respectively. The fourth component corresponds to diquark-antidiquark (tetraquark) bound states. The resulting frame-independent color-confining bound-state LF eigensolutions can be identified with the hadronic eigenstates of confined quarks for $\mathrm{SU}(3)$ color. In effect, two of the quarks of the baryonic color-singlet $q 9 q$ bound state bind to a color $\overline{3_{C}}$ diquark bound state, which then binds by the same color force to the remaining $3_{C}$ quark. As shown by t'Hooft in a string model [20], the $Y$ configuration of three quarks is unstable and reduces to the quark-diquark configuration. The matching of the meson and baryon spectra is thus due to the fact that the same color-confining potential that binds two quarks to a diquark also binds a quark to an antiquark.

Note that the same slope controls the Regge trajectories of both mesons and baryons in both the orbital angular momentum $L$ and the principal quantum number $n$. Only one mass parameter $\kappa=\omega^{2}$ appears; it sets the confinement scale and the hadron mass scale in the chiral limit, as well as the length scale which underlies hadron structure. We will also use the notation $\lambda=\kappa^{2}$. In addition to the meson and baryon eigenstates, one also predicts color-singlet tetraquark diquark-antidiquark bound states with the same mass as the baryon.
The LF Schrödinger equations for baryons and mesons derived from superconformal algebra are shown in Figure 2. As explained above, the baryons on the proton (Delta) trajectory are bound states of a quark with color $3_{C}$ and scalar (vector) diquark with color $\overline{3}_{C}$. The proton eigenstates labeled $\psi^{+}$(parallel quark and baryon spins) and $\psi^{-}$(antiparallel quark and baryon spins) have equal Fock state probability, a feature of "quark chirality invariance." Predictions for the static properties of the nucleons are discussed in [21].

Superconformal algebra also predicts that the LFWFs of the superpartners are related, and thus the corresponding elastic and transition form factors are identical. The resulting predictions for meson and baryon timelike form factors can be tested in $e^{+} e^{-} \rightarrow H \bar{H}^{\prime}$ reactions.

One can generalize these results to heavy-light $[\bar{Q} q]$ mesons and $[Q[q q]]$ baryons [22]. The Regge slopes are found to increase for heavy $m_{Q}$ as expected from heavy quark effective field theory; however, the supersymmetric connections between the heavy-light hadrons are predicted to be maintained.

The LFWFs thus play the same role in hadron physics as the Schrödinger wavefunctions which encode the structure of atoms in QED. The elastic and transition form factors of hadrons, weak-decay amplitudes, and distribution amplitudes are overlaps of LFWFs; structure functions, transverse momentum distributions, and other inclusive observables are constructed from the squares of the LFWFs. In contrast one cannot compute form factors of hadrons or other current matrix elements of hadrons from overlap of the usual "instant" form wavefunctions since one must also include contributions where the photon interacts with connected but acausal vacuum-induced currents. The calculation of deeply virtual Compton scattering using LFWFs is given in [23]. One 


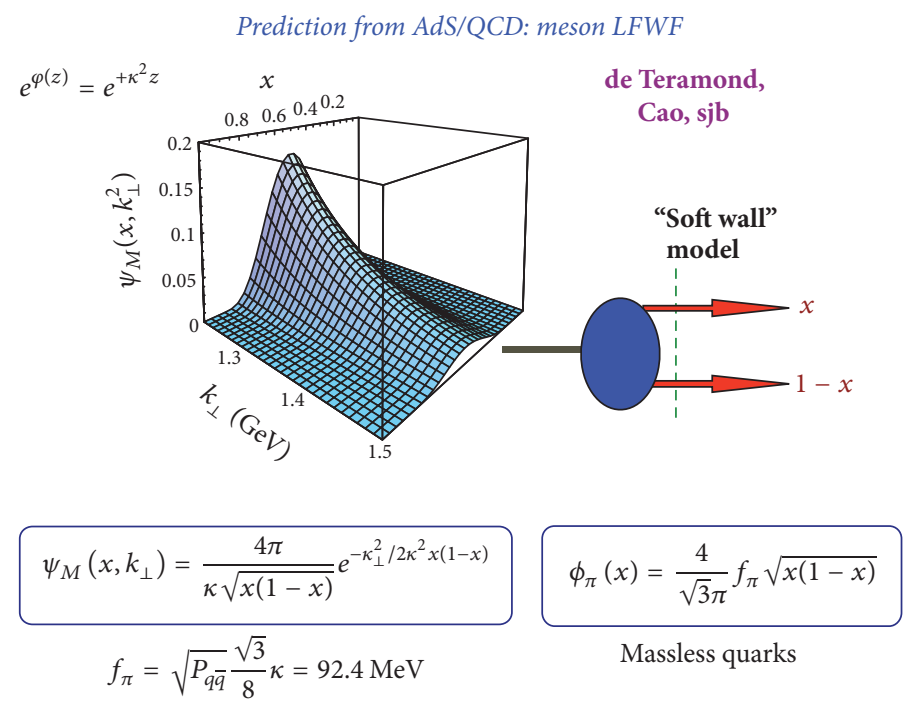

Figure 5: Prediction from AdS/QCD and Light-Front Holography for meson LFWFs $\psi_{M}\left(x, \vec{k}_{\perp}\right)$ and the pion distribution amplitude.

can also compute the gravitational form factors of hadrons. In particular, one can show that the anomalous gravitomagnetic moment $B\left(q^{2}=0\right)$ vanishes identically for any LF Fock state [24], in agreement with the equivalence theorem of gravity $[25,26]$.

The hadronic LFWFs predicted by light-front holography and superconformal algebra are functions of the LF kinetic energy $\vec{k}_{\perp}^{2} / x(1-x)$, the conjugate of the LF radial variable $\zeta^{2}=b_{\perp}^{\frac{1}{2}} x(1-x)$, times a function of $x(1-x)$. The hadronic LFWFs cannot be factorized as a function of $\vec{k}_{\perp}^{2}$ times a function of $x$. The resulting nonperturbative pion distribution amplitude $\phi_{\pi}(x)=\int d^{2} \vec{k}_{\perp} \psi_{\pi}\left(x, \vec{k}_{\perp}\right)=$ $(4 / \sqrt{3} \pi) f_{\pi} \sqrt{x(1-x)}$, presented in Figure 5 , which controls hard exclusive process, is consistent with the Belle data for the photon-to-pion transition form factor [27]. The AdS/QCD light-front holographic eigenfunction for the $\rho$ meson LFWF $\psi_{\rho}\left(x, \vec{k}_{\perp}\right)$ gives excellent predictions for the observed features of diffractive $\rho$ electroproduction $\gamma^{*} p \rightarrow$ $\rho p^{\prime}$, as shown by Forshaw and Sandapen [28].

\section{Light-Front Holography}

Five-dimensional $\mathrm{AdS}_{5}$ space provides a geometrical representation of the conformal group. The color-confining lightfront equation for mesons of arbitrary spin $J$ can be derived [4] from the holographic mapping of the "soft-wall model" modification of $\mathrm{AdS}_{5}$ space for the specific dilaton profile $e^{+\kappa^{2} z^{2}}$, where one identifies the fifth dimension coordinate $z$ with the light-front coordinate $\zeta$. Remarkably, $\mathrm{AdS}_{5}$ is holographically dual to $3+1$ space-time at fixed light-front time $\tau=t+z / c$. The holographic dictionary is summarized in Figure 6. The combination of light-front dynamics, its holographic mapping to $\mathrm{AdS}_{5}$ space, and the dAFF procedure provides new insight into the physics underlying color confinement, the nonperturbative QCD coupling, and the

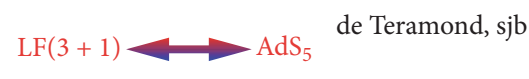

Light-Front Holographic Dictionary

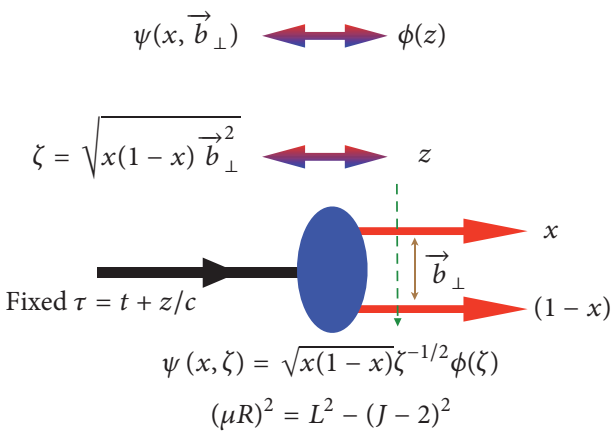

Light-front holography: unique mapping derived from equality of $L F$ and AdS formula for EM and gravitational current matrix elements and identical equations of motion

FIGURE 6: The holographic dictionary which maps the fifth dimension variable $z$ of the five-dimensional $\mathrm{AdS}_{5}$ space to the LF radial variable $\zeta$ where $\zeta^{2}=b_{\perp}^{2}(1-x)$. The same physics transformation maps the $\mathrm{AdS}_{5}$ and $(3+1) \mathrm{LF}$ expressions for electromagnetic and gravitational form factors to each other, from [4].

QCD mass scale. A comprehensive review is given in [29]. The $q \bar{q}$ mesons and their valence LF wavefunctions are the eigensolutions of the frame-independent relativistic bound state LF Schrödinger equation, the same meson equation that is derived using superconformal algebra. The mesonic $q \bar{q}$ bound-state eigenvalues for massless quarks are $M^{2}(n, L, S)=$ $4 \kappa^{2}(n+L+S / 2)$. The equation predicts that the pion eigenstate $n=L=S=0$ is massless at zero quark mass. The Regge spectra of the pseudoscalar $S=0$ and vector $S=1$ mesons are predicted correctly, with equal slope in the principal quantum number $n$ and the internal orbital angular momentum $L$. A comparison with experiment is shown in Figure 7. 

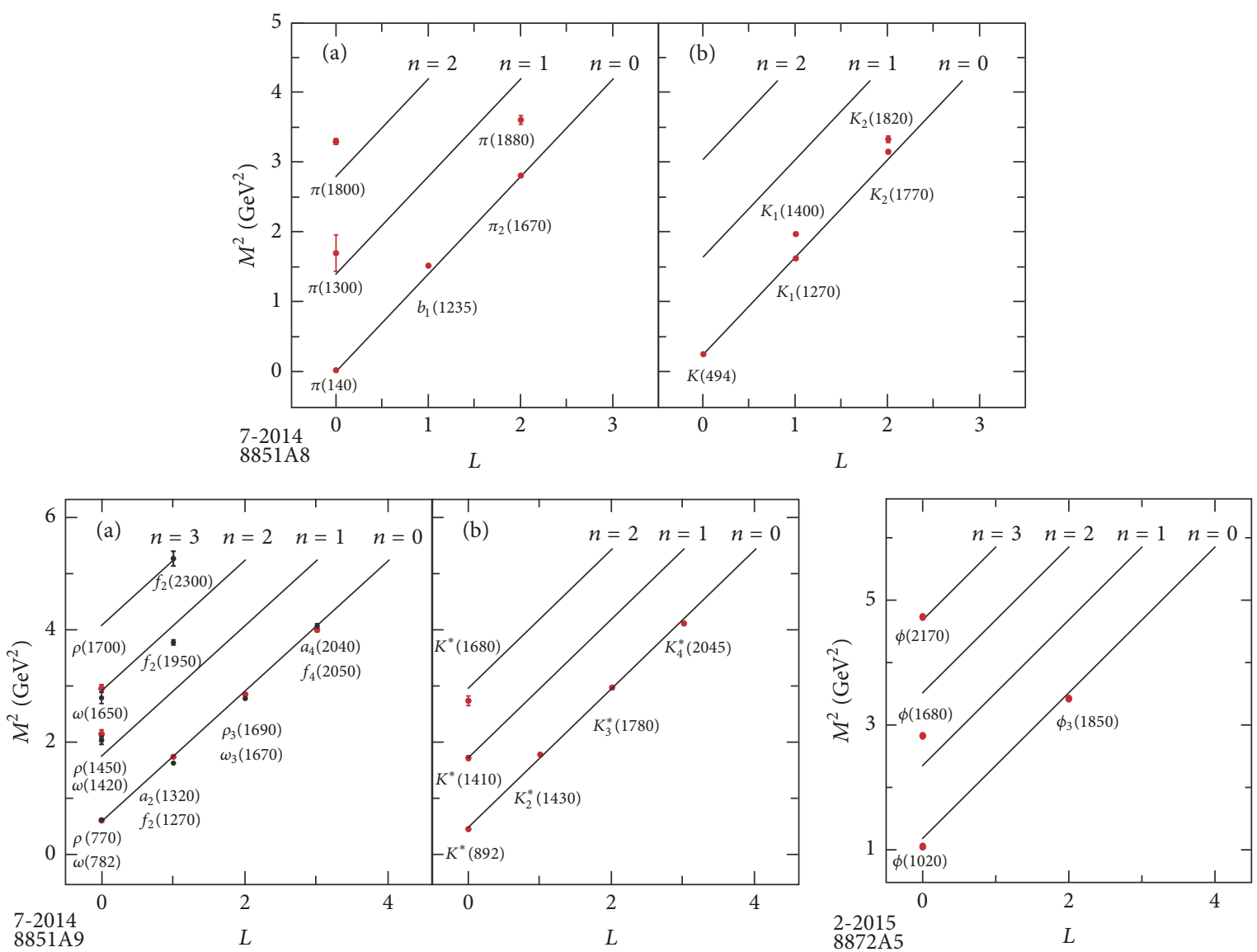

Figure 7: Comparison of the AdS/QCD prediction $M^{2}(n, L, S)=4 \kappa^{2}(n+L+S / 2)$ for the orbital $L$ and radial $n$ excitations of the meson spectrum with experiment. The pion is predicted to be massless for zero quark mass. The $u, d, s$ quark masses can be taken into account by perturbing in $\left\langle m_{q}^{2} \mid x\right\rangle$. The fitted value of $\kappa=0.59 \mathrm{GeV}$ for pseudoscalar mesons, and $\kappa=0.54 \mathrm{GeV}$ for vector mesons.

Light-Front Holography predicts not only meson and baryon spectroscopy successfully, but also hadron dynamics, including vector meson electroproduction, hadronic lightfront wavefunctions, distribution amplitudes, form factors, and valence structure functions. The application to the deuteron elastic form factors and structure functions is given in $[30,31]$.

\section{Color Confinement from LF Holography}

Remarkably, the light-front potential using the dAFF procedure has the unique form of a harmonic oscillator $\kappa^{4} \zeta^{2}$ in the light-front invariant impact variable $\zeta$ where $\zeta^{2}=b_{\perp}^{2} x(1-x)$. The result is a single-variable frame-independent relativistic equation of motion for $q \bar{q}$ bound states, a "Light-Front Schrödinger Equation" [32], analogous to the nonrelativistic radial Schrödinger equation in quantum mechanics. The same result, including spin terms, is obtained using lightfront holography-the duality between the front form and $\mathrm{AdS}_{5}$, the space of isometries of the conformal group-if one modifies the action of $\mathrm{AdS}_{5}$ by the dilaton $e^{\kappa^{2} z^{2}}$ in the fifth dimension $z$. The Light-Front Schrödinger Equation incorporates color confinement and other essential spectroscopic and dynamical features of hadron physics, including a massless pion for zero quark mass and linear Regge trajectories with the same slope in the radial quantum number $n$ and internal orbital angular momentum $L$. When one generalizes this procedure using superconformal algebra, the resulting light-front eigensolutions predict a unified Regge spectroscopy of meson, baryon, and tetraquarks, including remarkable supersymmetric relations between the masses of mesons and baryons of the same parity.

It is interesting to note that the contribution of the " $H$ " diagram to $Q \bar{Q}$ scattering is IR divergent as the transverse separation between $Q$ and $\bar{Q}$ increases [33]. This is a signal that $\mathrm{pQCD}$ is inconsistent without color confinement. The sum of such diagrams could sum to the confinement potential $\kappa^{4} \zeta^{2}$ dictated by the dAFF principle that the action remains conformally invariant despite the appearance of the mass scale $\kappa$ in the Hamiltonian. The $\kappa^{4} \zeta^{2}$ confinement interaction between a $q$ and $\bar{q}$ will induce a $\kappa^{4} / s^{2}$ correction to $R_{e^{+} e^{-}}$, replacing the $1 / s^{2}$ signal usually attributed to a vacuum gluon condensate.

It should be emphasized that the value of the mass scale $\kappa$ is not determined in an absolute sense by QCD. Only ratios of masses are determined, and the theory has dilation invariance under $\kappa \rightarrow C \kappa$. In a sense, chiral QCD has an "extended conformal invariance." The resulting new time 


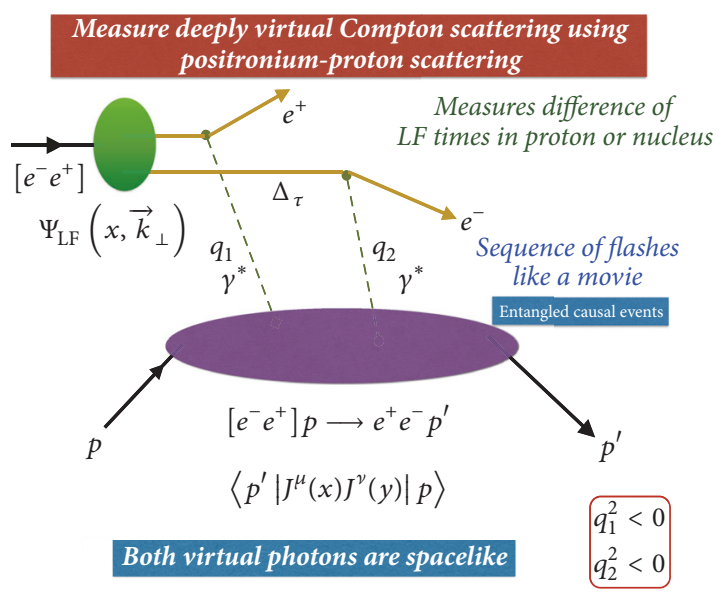

FIGURE 8: Doubly virtual Compton scattering on a proton (or nucleus) can be measured for two spacelike photons $q_{1}^{2}, q_{2}^{2}<0$ with minimal, tunable, skewness $\xi$ using positronium-proton scattering $\left[e^{+} e^{-}\right] p \rightarrow e^{+} e^{-} p^{\prime}$. One can also measure double deep inelastic scattering and elastic positronium-proton scattering. One can also create a beam of "true muonium" atoms $\left[\mu^{-} \mu^{-}\right][5,6]$ using BetheHeitler pair production just below threshold.

variable, which retains the conformal invariance of the action, has finite support, conforming to the fact that the LF time between the interactions with the confined constituents is finite. The finite time difference $\Delta \tau$ between the LF times $\tau$ of the quark constituents of the proton could be measured using positronium-proton scattering $\left[e^{+} e^{-}\right] p \rightarrow e^{+} e^{-} p^{\prime}$. This process, which measures double diffractive deeply virtual Compton scattering for two spacelike photons, is illustrated in Figure 8. One can also study the dissociation of relativistic positronium atoms to an electron and positron with light-front momentum fractions $x$ and $1-x$ and opposite transverse momenta in analogy to the E791 measurements of the diffractive dissociation of the pion to two jets [34]. The LFWF of positronium in the relativistic domain is the central input. One can produce a relativistic positronium beam using the collisions of laser photons with high energy photons or by using Bethe-Heitler pair production below the $e^{+} e^{-}$threshold. The production of parapositronium via the collision of photons is analogous to pion production in twophoton interactions and Higgs production via gluon-gluon fusion.

\section{Light-Front Theory and QCD}

One can derive the exact form of the light-front Hamiltonian $H_{\mathrm{LF}}$ directly from the QCD Lagrangian and avoid ghosts and longitudinal gluonic degrees of freedom by choosing the light-cone gauge $A^{+}=0$. Quark masses appear in the LF kinetic energy as $\sum_{i}\left(m^{2} / x_{i}\right)$. This can be derived from the Higgs theory quantized using LF dynamics [35]. The confined quark field $\psi_{q}$ couples to the background Higgs field $g_{\bar{\Psi}_{q}}\langle H\rangle \Psi_{q}$ via its Yukawa scalar matrix element coupling $g_{q}\langle H\rangle \bar{u}(p) 1 u(p)=m_{q} \times\left(m_{q} / x\right)=m^{2} / x$. The usual Higgs vacuum expectation value $\langle H\rangle$ is replaced by a constant zero mode when one quantizes the Standard Model using lightfront quantization [35].

PQCD factorization theorems and the DGLAP [36-38] and ERBL [39-42] evolution equations can also be derived using the light-front Hamiltonian formalism [40]. In the case of an electron-ion collider, one can represent the crosssection for $e-p$ collisions as a convolution of the hadron and virtual photon structure functions times the subprocess cross-section in analogy to hadron-hadron collisions. This nonstandard description of $\gamma^{*} p \rightarrow X$ reactions gives new insights into electroproduction physics, physics not apparent in the usual infinite-momentum frame description, such as the dynamics of heavy quark-pair production. Intrinsic heavy quarks at high $x$ also play an important role [43].

The LF Heisenberg equation can in principle be solved numerically by matrix diagonalization using the "Discretized Light-Cone Quantization" (DLCQ) [44] method. Antiperiodic boundary conditions in $x^{-}$render the $k^{+}$momenta discrete as well as limiting the size of the Fock basis. In fact, one can easily solve $1+1$ quantum field theories such as QCD $(1+1)$ [45] for any number of colors, flavors, and quark masses using DLCQ. Unlike lattice gauge theory, the nonperturbative DLCQ analysis is in Minkowski space, is frame-independent, and is free of fermion-doubling problems. AdS/QCD, based on the $\mathrm{AdS}_{5}$ representation of the conformal group in five dimensions, maps to physical $3+$ 1 space-time at fixed LF time; this correspondence, "lightfront holography" [32], is now providing a color-confining approximation to $H_{\mathrm{LF}}^{\mathrm{QCD}}$ for $\mathrm{QCD}(3+1)$. This method gives a remarkable first approximation to hadron spectroscopy and hadronic LFWFs. A new method for solving nonperturbative QCD “Basis Light-Front Quantization” (BLFQ) [46], uses the eigensolutions of a color-confining approximation to QCD (such as LF holography) as the basis functions, rather than the plane-wave basis used in DLCQ, thus incorporating the full dynamics of QCD. LFWFs can also be determined from the covariant Bethe-Salpeter wavefunction by integrating over $k^{-}$ [47]. A review of the light-front formalism is given in [14].

\section{Measuring LFWFs of Hadrons, Atoms, and Nuclei}

One can in fact measure the LFWFs of QED atoms using diffractive dissociation.

For example, suppose one creates a relativistic positronium beam. It will dissociate by Coulomb exchange in a thin target: $\left[e^{+} e^{-}\right]+Z \rightarrow e^{+} e^{-} Z$. The momentum distribution of the leptons in the LF variables $x$ and $k_{\perp}$ will determine the first derivative of the atomic $\operatorname{LFWF}\left(d / d k_{\perp}\right) \psi\left(x, \vec{k}_{\perp}\right)$. When $k_{\perp}^{2} / x(1-x)>4 m_{e}^{2}$ one can observe the transition from NR Schrödinger theory where $\psi\left(x, \vec{k}_{\perp}\right) \propto 1 / k_{\perp}^{4}$ to the relativistic domain, where $\psi\left(x, \vec{k}_{\perp}\right) \propto 1 / k_{\perp}^{2}$. One can thus test predictions from BLFQ (Basis Light-Front Quantization) [48]. Higher Fock states are also possible, such as $\left[e^{+} e^{-}\right]+$ $Z \rightarrow e^{+} e^{-} \gamma Z$ and $\left[e^{+} e^{-}\right]+Z \rightarrow e^{+} e^{-} e^{+} e^{-} Z$ ?

Positronium dissociation is analogous to the Ashery measurements of the pion LFWF: $\pi A \rightarrow \operatorname{JetJet} A$ [49], where 


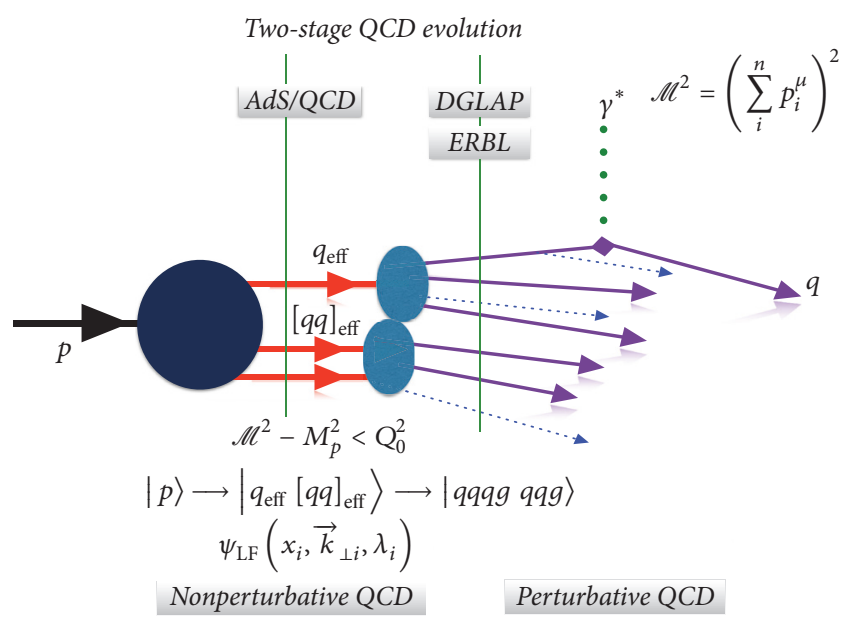

(a)

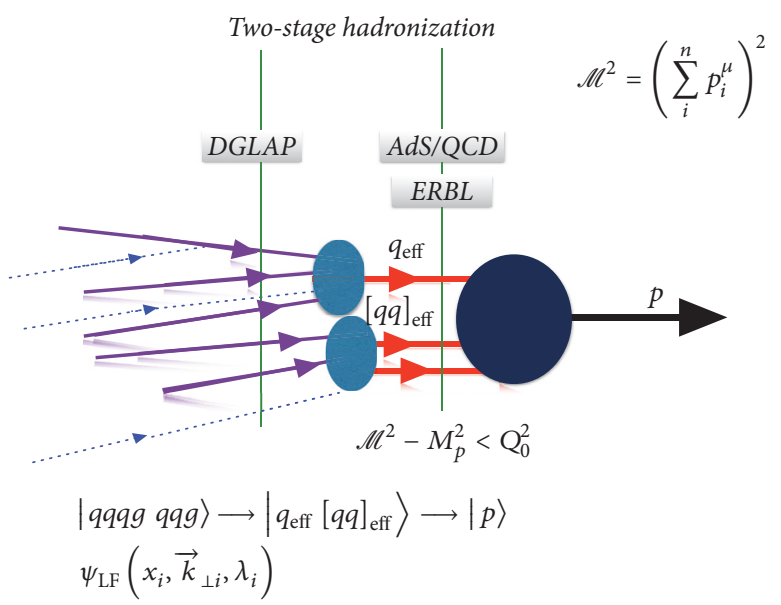

(b)

FIGURE 9: (a) A model for evolution starting with a nonperturbative hadronic LFWF. (b) Hadronization and evolution ending with a hadronic LFWF. The intermediate quark and gluon states are off the $P^{-}$energy shell and thus off-the-invariant mass shell $\mathscr{M}^{2}>m_{H}^{2}$. In the off-shell domain $\mathscr{M}^{2}-M_{H}^{2}>\kappa^{2}$, the intermediate quarks and gluons obey the DGLAP and ERBL QCD evolution. If a cluster of quarks and antiquarks satisfies $\mathscr{M}^{2}-M_{H}^{2}<\kappa^{2}$, the intermediate state sees the color confinement interaction. The meson LFWF connects the intermediate $q \bar{q}$ state, which is off of the $P^{-}$energy shell and thus off-the-invariant mass shell $\mathscr{M}^{2}>m_{H}^{2}$ to the physical meson state with $\mathscr{M}^{2}=m_{H}^{2}$. The LF angular momentum $J^{z}$ is conserved at every vertex.

one observes the transition from Gaussian fall-off to power law fall-off at large $1 / k_{\perp}^{2}$ as predicted by AdS/QCD. When $k_{\perp}^{2} / x(1-x)>4 m_{e}^{2}$ one can measure the transition from NR Schrödinger theory to the relativistic domain, where $\psi\left(x, \vec{k}_{\perp}\right) \propto 1 / k_{\perp}^{2}$. Similarly, one could also measure the LFWF of a nucleus like a deuteron by dissociating relativistic ions $d A \rightarrow p n A$. At large $1 / k_{\perp}^{2}$ one could observe the transition to the "hidden-color" Fock states predicted by QCD [50].

\section{Calculations Using LF-Time-Ordered Perturbation Theory and Hadronization at the Amplitude Level}

LF-time-ordered perturbation theory can be advantageous for perturbative QCD calculations. An excellent example of LF-time-ordered perturbation theory is the computation of multigluon scattering amplitudes by Cruz-Santiago et al. [51]. In this method, the propagating particles are on their respective mass shells: $k_{\mu} k^{\mu}=m^{2}$, and intermediate states are off-shell in invariant mass; that is, $P^{-} \neq \sum k_{i}^{-}$. Unlike instant form, where one must sum $n$ ! frame-dependent amplitudes, only the $\tau$-ordered diagrams where each propagating particle has positive $k^{+}=k^{0}+k^{z}$ can contribute. The number of nonzero amplitudes is also greatly reduced by noting that the total angular momentum projection $J^{z}=\sum_{i}^{n-1} L_{i}^{z}+\sum_{i}^{n} S_{i}^{z}$ and the total $P^{+}$are conserved at each vertex. In a renormalizable theory, the change in orbital angular momentum is limited to $\Delta L^{z}=0, \pm 1$ at each vertex [52].

A remarkable advantage of LF time-ordered perturbation theory (LFPth) is that the calculation of a subgraph of any order in PQCD only needs to be done once; the result can be stored in a "history" file. This is due to the fact that in LFPth the numerator algebra is independent of the process; the denominator changes, but only by a simple shift of the initial $P^{-}$. Another simplification is that loop integrations are three dimensional: $\int d^{2} \vec{k} \perp \int_{0}^{1} d x$. Unitarity and explicit renormalization can be implemented using the "alternate denominator" method which defines the required subtraction counterterms [53].

The new insights into color confinement given by AdS/QCD suggest that one could compute "hadronization at the amplitude level" [54] using the confinement interaction and the LFWFs predicted by AdS/QCD and Light-Front Holography. For example, as illustrated in Figure 1, the meson LFWF connects the off-the-invariant mass shell quark and antiquark to the on-shell asymptotic physical meson state.

The invariant mass of a color-singlet cluster $\mathscr{M}$ is the key variable which separates perturbative and nonperturbative dynamics. For example, consider $e^{+} e^{-}$annihilation using LF $\tau$-ordered perturbation theory. At an early stage in LF time, the annihilation will produce jets of quarks and gluons in an intermediate state off the $P^{-}$energy shell. If a color-singlet cluster of partons in a jet satisfies $\mathscr{M}^{2}-M_{H}^{2}<\kappa^{2}$, the cluster constituents are effective degrees of freedom which will be ruled by the $\kappa^{4} \zeta^{2}$ color-confinement potential. At this stage, the LFWF $\psi_{H}$ converts the off-shell partons to the asymptotic states, the on-shell hadrons. If $\mathscr{M}^{2}>\kappa^{2}$ one can apply pQCD corrections, for example, from DGLAP and ERBL evolution [39-42].

A model for the two stages of hadronization and evolution is illustrated in Figure 9. In the off-shell domain $\mathscr{M}^{2}-M_{H}^{2}>$ $\kappa^{2}$, the intermediate quarks and gluons obey DGLAP and ERBL evolution.

Thus quarks and gluons can appear in intermediate offshell states, but only hadrons are produced asymptotically. 
Thus the AdS/QCD Light-Front Holographic model suggests how one can implement the transition between perturbative and nonperturbative QCD. For a QED analog, see $[5,6]$.

\section{Light-Front Spin and Light-Front $J^{z}$ Conservation}

A central, unique property of light-front quantization is $J^{z}$ conservation [52]; the $z$-component of angular momentum remains unchanged under Lorentz transformations generated by the light-front kinematical boost operators. The spin along the $\widehat{z}$ direction defined by the light-front Lorentz transformation is preserved because $\left\langle J^{3}\right\rangle_{\mathrm{LF}}=s^{z}$ for all momenta $p^{\mu}$. $J^{z}$ conservation underlies the Jaffe spin sum rule [55].

Particles in the front form move with positive $k^{+}=k^{0}+$ $k^{z} \geq 0$. The quantization axis for $J^{z}$ for each particle is the same axis $\widehat{z}$ which defines LF time $\tau=t+z / c$. Thus $S^{z}$ and $L^{z}$ refer to angular momentum in the $\widehat{z}$ direction. As in nonrelativistic quantum mechanics, $J^{z}=\sum_{i=1}^{n} S_{i}^{z}+$ $\sum_{n=1}^{n-1} L_{i}^{z}$ for any $n$-particle intermediate or Fock state. There are $n-1$ relative orbital angular momenta. It is conserved at every vertex and is conserved overall for any process and "LF helicity" refers to the spin projection $S^{z}$ of each particle and "LF chirality" is the spin projection $S^{z}$ for massless particles. In a renormalizable theory $L^{z}$ can only change by one unit at any vertex. This leads to a rigorous selection rule for amplitudes at fixed order in pQCD [52]: $\left|\Delta L^{z}\right| \leq n$ in an $n$th order perturbative expansion. This selection rule for the orbital angular momentum can be used to eliminate interaction vertices in QED and QCD and provides an upper bound on the change of orbital angular momentum in scattering processes at any fixed order in perturbation theory.

By definition, spin and helicity can be used interchangeably in the front form. LF chirality is conserved by the vector current in electrodynamics and the axial current of electroweak interactions. Each coupling conserves quark chirality when the quark mass is set to zero. A compilation of LF spinor matrix elements is given in [40].

Light-front spin is not the same as the usual "Wick helicity," where spin is defined as the projection of the particle's three-momentum $\vec{k}$. Wick helicity is thus not conserved unless all particles move in the same direction. Wick helicity can be frame dependent. For example, In the case of $g g \rightarrow H$, the Wick helicity assignment is $(+1)+(+1) \rightarrow 0$ in the CM frame, but it is $(+1)+(-1) \rightarrow 0$ for collinear gluons if the two gluons move in the same direction.

The twist of a hadronic interpolating operator corresponds to the number of fields plus $\left|L^{z}\right|$. The pion LF Fock state for $\pi \rightarrow q \bar{q}$ with twist-2 corresponds to $\left(J_{\pi}^{z}=0\right) \rightarrow$ $\left(S_{q}^{z}=+1 / 2\right)+\left(S^{z}=-1 / 2\right)$ with zero relative orbital angular momentum $L_{q \bar{q}}^{z}$. This is the Fock state of the pion that decays to $\ell v$ via the LF chiral-conserving axial current $\gamma^{\mu} \gamma_{5}$. The twist-3 pion in the OPE corresponds to $J_{\pi}^{z}=0 \rightarrow\left(S_{q}^{z}=\right.$ $+1 / 2)+\left(S_{\bar{q}}^{z}=+1 / 2\right)+\left(L^{z}=-1\right)$ or $J^{z}=0 \rightarrow\left(S_{q}^{z}=\right.$ $-1 / 2)+\left(S_{\bar{q}}^{z}=-1 / 2\right)+\left(L_{q \bar{q}}^{z}=-1\right)$, where $L^{z}$ is the relative orbital angular momentum between the quark and antiquark.
The twist-3 Fock state couples the pion to the chiral-flip pseudoscalar $\gamma_{5}$ operator. The GMOR relation connects the twist-2 and twist-3 Fock states when $m_{q} \neq 0$ [56]. The twist3 proton with $J_{p}^{z}=+1 / 2$ in AdS/QCD is a bound state of a quark with $S_{p}^{z}=1 / 2$ and a spin-zero diquark [qq] with $L_{q[q q]}^{z}=0$, and the twist-4 proton in AdS/QCD is a bound state of a quark with $S_{p}^{z}=-1 / 2$ and spin-zero diquark [qq] with relative orbital angular momentum $L_{q[q q]}^{z}=+1$. LF holography predicts equal probability for the twist- 3 and twist-4 Fock states in the nucleon for $m_{q}=0$.

One can use LF $J^{z}$ conservation to determine the contribution of Fock states of different twist in a scattering amplitude by using the fact that amplitudes with nonzero relative $L^{z}$ between the outgoing particles vanish in the forward direction. For example, consider pion electroproduction $\gamma^{*} p \rightarrow \pi^{0} p$ for a polarized photon with LF spin $S_{\gamma}^{z}=-1$. If the proton's LF spin $S_{p}^{z}=-1 / 2$ is unchanged, then $J_{\text {tot }}^{z}=+1 / 2: \gamma_{T}^{*}\left(S_{\gamma}^{z}=+1\right)+\left(S_{p}^{z}=-1 / 2\right) \rightarrow+\left(J_{\pi}^{z}=\right.$ $0)+\left(S_{p}^{z}=-1 / 2\right)+\left(L_{\pi^{0}-p}^{z}=+1\right)$ vanishes at $t=0$ for the twist-2 pion. However, the non-spin-flip proton amplitude $J_{\text {tot }}^{z}=1 / 2: \gamma_{T}^{*}\left(S_{\gamma}^{z}=+1\right)+S_{p}^{z}=(-1 / 2) \rightarrow\left[\left(S_{q}^{z}=\right.\right.$ $\left.-1 / 2)+\left(S_{\bar{q}}^{z}=-1 / 2\right)+\left(L_{q \bar{q}}^{z}=+1\right)\right]_{\pi^{0}}+S_{p}^{z}=(-1 / 2)$ for the twist-3 pion Fock state is finite at $t=0$. A similar result holds for the contribution of the twist- 2 pion and twist- 4 proton. See Figure 10.

Similarly one can utilize the behavior of the amplitude $\gamma^{*} \mathrm{He}^{4} \rightarrow \pi^{0} \mathrm{He}^{4}$ on a spin-zero helium target. The pion twist2 amplitude with $J_{\text {tot }}^{z}=+1: \gamma_{T}^{*}\left(S_{\gamma}^{z}=+1\right)+\left(S_{\mathrm{He}}^{z}=0\right) \rightarrow\left[\left(S_{q}^{z}=\right.\right.$ $\left.+1 / 2)+\left(S_{\bar{q}}^{z}=-1 / 2\right)+\left(L_{q \bar{q}}^{z}=0\right)\right]_{\pi^{0}}+\left(S_{\mathrm{He}}^{z}=0\right)+\left(L_{\pi^{0} \mathrm{He}^{4}}^{z}=1\right)$ vanishes at $t=0$, whereas the amplitude with a pion twist3 amplitude $J_{\text {tot }}^{z}=+1: \gamma_{T}^{*}\left(S_{\gamma}^{z}=+1\right)+\left(S_{\mathrm{He}}^{z}=0\right) \rightarrow\left[\left(S_{q}^{z}=\right.\right.$ $\left.+1 / 2)+\left(S_{\bar{q}}^{z}=-1 / 2\right)+\left(L_{q \bar{q}}^{z}=+1\right)\right]_{\pi^{0}}+\left(S_{\mathrm{He}}^{z}=0\right)+\left(L_{\pi^{0} \mathrm{He}^{4}}^{z}=0\right)$ is finite at $t=0$, thus discriminating between contributions using the twist- 2 and twist- 3 pion amplitudes.

\section{The Light-Front Vacuum}

It is important to distinguish the LF vacuum from the conventional instant-form vacuum. The eigenstates of the instantform Hamiltonian describe a state defined at a single instant of time $t$ over all space, and they are thus acausal as well as frame-dependent. The instant-form vacuum is defined as the lowest energy eigenstate of the instant-form Hamiltonian. As discussed by Zee [57], the cosmological constant is of order $10^{120}$ times larger than what is observed if one computes the effects of quantum loops from QED. Similarly, QCD instantons and condensates in the instant-form vacuum give a contribution of order $10^{42}$. The contribution of the Higgs VEV computed in the instant-form vacuum is $10^{54}$ times too large.

In contrast, the vacuum in LF Hamiltonian theory is defined as the eigenstate of $H_{\mathrm{LF}}$ with lowest invariant mass. It is defined at fixed LF time $\tau$ within the causal horizon, and it is frame-independent; that is, it is independent of the observer's motion. Vacuum loop diagrams from quantum field theory do not appear in the front-form vacuum since the + momenta 


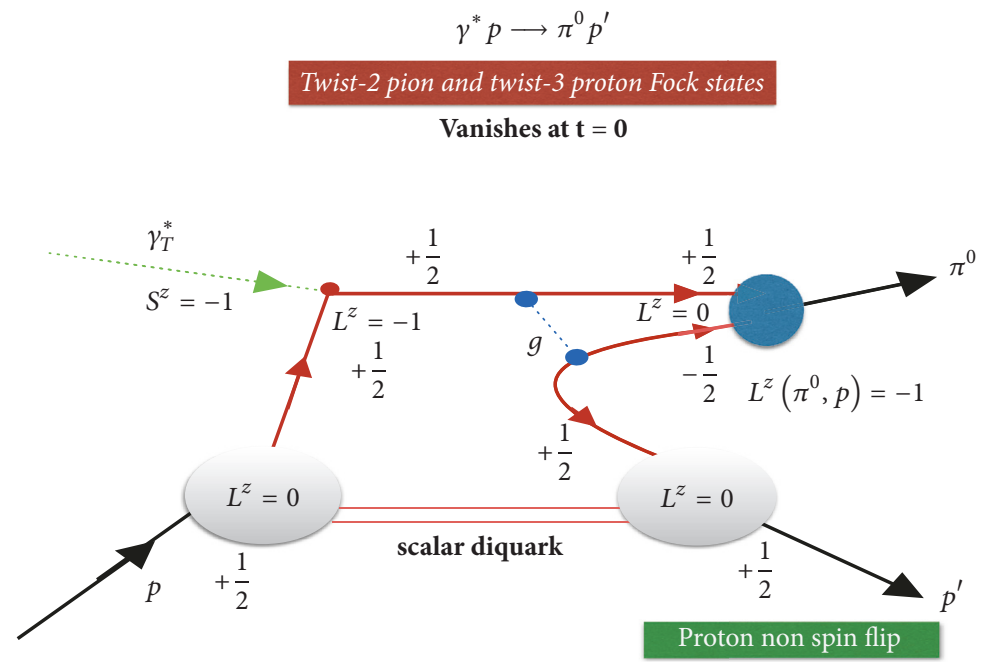

$$
\gamma^{*} p \longrightarrow \pi^{0} p^{\prime}
$$

Uses twist-3 pion and twist-3 proton Fock states

Finite at $\mathbf{t}=\mathbf{0}$

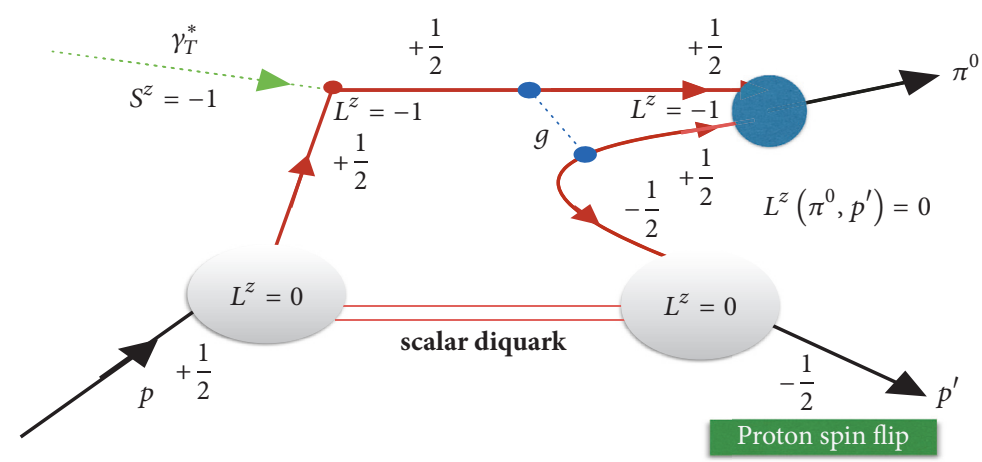

$$
\gamma^{*} p \longrightarrow \pi^{0} p^{\prime}
$$

\section{Twist-2 pion and twist-4 proton Fock states}

Finite at $\mathbf{t}=\mathbf{0}$

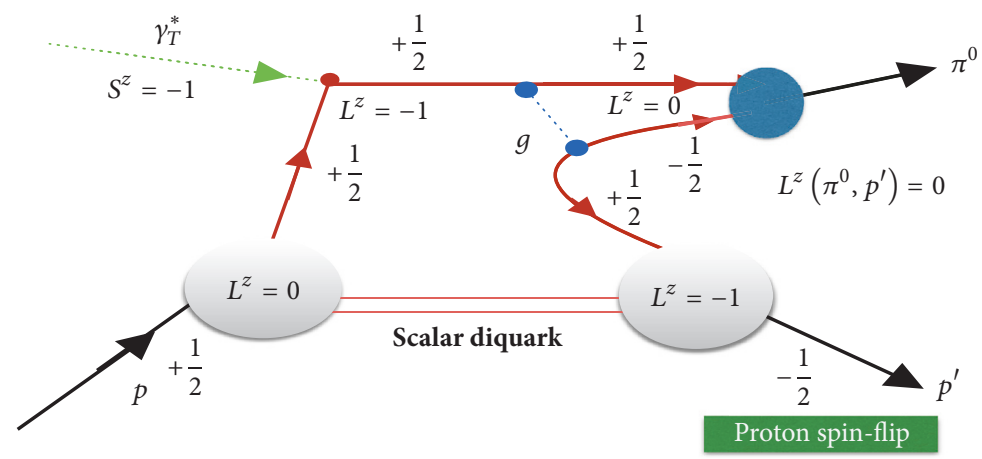

Figure 10: Illustration of spin flow in $\gamma^{*} p \rightarrow \pi^{0} p$. 
are positive: $k_{i}^{+}=k_{i}^{0}+k_{i}^{z} \geq 0$, and the sum of + momenta is conserved at every vertex. The creation of particles cannot arise from the LF vacuum since $\sum_{i} k^{+i} \neq P_{\text {vacuum }}^{+}=0$. Propagation with negative $k^{+}$does not appear. The physical vacuum state can also have $k^{+}=0$ modes corresponding to a flat energy-momentum background, analogous to a classical scalar Stark or Zeeman field. For example, Reinhardt and Weigel [58] have shown that the Nambu-Jona-Lasino (NJL) model can lead to a nontrivial physical LF vacuum. In the case of the Higgs theory, the traditional Higgs vacuum expectation value (VEV) is replaced by a "zero mode" [35]. All phenomenological consequences of the Higgs theory in the Standard Model are unchanged in the LF formulation. As noted in Section 6, the $\mathrm{m}^{2} / x$ term in the LF kinetic energy $\left(k_{\perp}^{2}+m^{q}\right) / x$ arises from the interaction of a quark within a hadron in QCD with its Yukawa interaction with the Higgs background zero mode.

The physics associated with quark and gluon QCD vacuum condensates of the instant form are replaced by physical effects contained within the hadronic LFWFs. This is referred to as "in-hadron" condensates [59-61]. For example, as discussed in Section 7, the GMOR relation relating the vacuum-to-pion matrix elements of the axial current and pseudoscalar operators is satisfied in LF theory as a relation between the twist- 2 and twist-3 Fock states [56]. The usual properties of chiral symmetry are satisfied; for example, as discussed in Section 2, the mass of the pion eigenstate computed from LF holography vanishes for zero quark mass.

The universe is observed within the causal horizon, not at a single instant of time. The causal, frame-independent light-front vacuum can thus provide a viable match to the empty visible universe [61]. The huge contributions to the cosmological constant from quantum field theory loops thus do not appear if one notes that the causal, frame-independent light-front vacuum has no quantum fluctuations, in dramatic contrast to the acausal, frame-dependent instant-form vacuum; the cosmological constant arising from quantum field theory thus vanishes if one uses the front form. The Higgs LF zero mode [35] has no energy-momentum density, so it also gives zero contribution to the cosmological constant. The observed nonzero value could be a property of gravity itself, such as the "emergent gravity" postulated by Verlinde [62]. It is also possible that if one solves electroweak theory in a curved universe, the Higgs LF zero mode would be replaced with a field of nonzero curvature which could give a nonzero contribution to the cosmological constant.

\section{The QCD Coupling at All Scales}

The QCD running coupling $\alpha_{s}\left(Q^{2}\right)$ sets the strength of the interactions of quarks and gluons as a function of the momentum transfer $Q$. The dependence of the coupling $Q^{2}$ is needed to describe hadronic interactions at both long and short distances. The QCD running coupling can be defined [63] at all momentum scales from a perturbatively calculable observable, such as the coupling $\alpha_{g_{1}}^{s}\left(Q^{2}\right)$, which is defined from measurements of the Bjorken sum rule. At high momentum transfer, such "effective charges" satisfy asymptotic freedom, obey the usual $\mathrm{pQCD}$ renormalization group equations, and can be related to each other without scale ambiguity by commensurate scale relations [64].

The dilaton $e^{+\kappa^{2} z^{2}}$ soft-wall modification of the $\mathrm{AdS}_{5}$ metric, together with LF holography, predicts the functional behavior of the running coupling in the small $Q^{2}$ domain [65]: $\alpha_{g_{1}}^{s}\left(Q^{2}\right)=\pi e^{-Q^{2} / 4 \kappa^{2}}$. Measurements of $\alpha_{g_{1}}^{s}\left(Q^{2}\right)$ are remarkably consistent [66] with this predicted Gaussian form; the best fit gives $\kappa=0.513 \pm 0.007 \mathrm{GeV}$. See Figure 11 . Brodsky et al. $[7,65,67]$ have also shown how the parameter $\kappa$, which determines the mass scale of hadrons and Regge slopes in the zero quark mass limit, can be connected to the mass scale $\Lambda_{s}$ controlling the evolution of the perturbative QCD coupling. The high momentum transfer dependence of the coupling $\alpha_{g 1}\left(Q^{2}\right)$ is predicted by pQCD. The matching of the high and low momentum transfer regimes of $\alpha_{g 1}\left(Q^{2}\right)$, both its value and its slope, then determines a scale $Q_{0}=$ $0.87 \pm 0.08 \mathrm{GeV}$ which sets the interface between perturbative and nonperturbative hadron dynamics. This connection can be done for any choice of renormalization scheme, such as the $\overline{\mathrm{MS}}$ scheme, as seen in Figure 11. The result of this perturbative/nonperturbative matching is an effective QCD coupling defined at all momenta. The predicted value of $\Lambda_{\overline{\mathrm{MS}}}=0.339 \pm$ $0.019 \mathrm{GeV}$ from this analysis agrees well the measured value [68] $\Lambda_{\overline{\mathrm{MS}}}=0.332 \pm 0.017 \mathrm{GeV}$. These results, combined with the AdS/QCD superconformal predictions for hadron spectroscopy, allow one to compute hadron masses in terms of $\Lambda_{\overline{\mathrm{MS}}}: m_{p}=\sqrt{2} \kappa=3.21 \Lambda_{\overline{\mathrm{MS}}}, m_{\rho}=\kappa=2.2 \Lambda_{\overline{\mathrm{MS}}}$, and $m_{p}=$ $\sqrt{2} m_{\rho}$, meeting a challenge proposed by Zee [69]. The value of $Q_{0}$ can be used to set the factorization scale for DGLAP evolution of hadronic structure functions and the ERBL evolution of distribution amplitudes. Deur, de Téramond, and I have also computed the dependence of $Q_{0}$ on the choice of the effective charge used to define the running coupling and the renormalization scheme used to compute its behavior in the perturbative regime. The use of the scale $Q_{0}$ to resolve the factorization scale uncertainty in structure functions and fragmentation functions, in combination with the schemeindependent principle of maximum conformality (PMC) [70] for setting renormalization scales, can greatly improve the precision of $\mathrm{PQCD}$ predictions for collider phenomenology.

\section{Is the Momentum Sum Rule Valid for Nuclear Structure Functions?}

Sum rules for deep inelastic scattering are usually analyzed using the operator product expansion of the forward virtual Compton amplitude, assuming it depends in the limit $Q^{2} \rightarrow$ $\infty$ on matrix elements of local operators such as the energymomentum tensor. The moments of structure functions and other distributions can then be evaluated as overlaps of the target hadron's light-front wavefunction, as in the Drell-YanWest formulae for hadronic form factors [71-74]. The real phase of the resulting DIS amplitude and its OPE matrix elements reflects the real phase of the stable target hadron's wavefunction. 


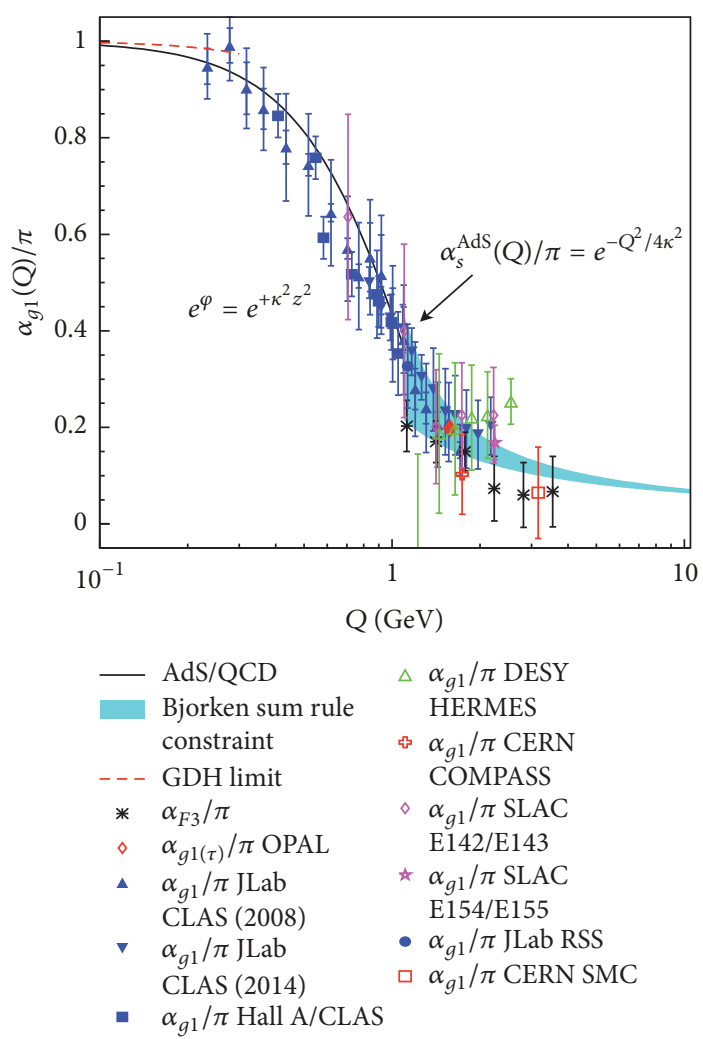

(a)

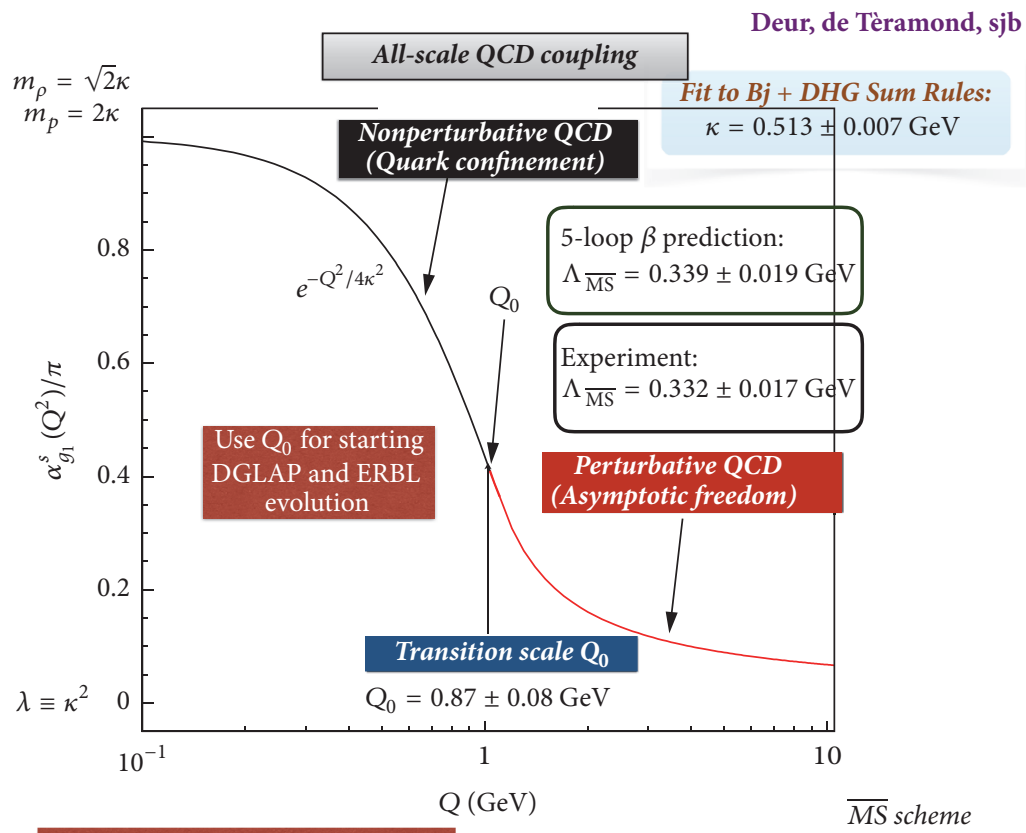

Reverse dimensional transmutation!

(b)

FIGURE 11: (a) Comparison of the predicted nonperturbative coupling, based on the dilaton $\exp \left(+\kappa^{2} z^{2}\right)$ modification of the AdS 5 metric, with measurements of the effective charge $\alpha_{g_{1}}^{s}\left(Q^{2}\right)$, as defined from the Bjorken sum rule. (b) Prediction from LF holography and pQCD for the QCD running coupling $\alpha_{g_{1}}^{s}\left(Q^{2}\right)$ at all scales. The magnitude and derivative of the perturbative and nonperturbative coupling are matched at the scale $Q_{0}$. This matching connects the perturbative scale $\Lambda_{\overline{\mathrm{MS}}}$ to the nonperturbative scale $\kappa$ which underlies the hadron mass scale. See [7]. 
The "handbag" approximation to deeply virtual Compton scattering also defines the "static" contribution $[75,76]$ to the measured parton distribution functions (PDF), transverse momentum distributions, and so on. The resulting momentum, spin, and other sum rules reflect the properties of the hadron's light-front wavefunction. However, the final-state interactions which occur after the lepton scatters on the quark can give nontrivial contributions to deep inelastic scattering processes at leading-twist and thus survive at high $Q^{2}$ and high $W^{2}=(q+p)^{2}$. For example, the pseudo-T-odd Sivers effect [77] is directly sensitive to the rescattering of the struck quark. Similarly, diffractive deep inelastic scattering (DDIS) involves the exchange of a gluon after the quark has been struck by the lepton [78]. In each case the corresponding DVCS amplitude is not given by the handbag diagram since interactions between the two currents are essential. These "lensing" corrections survive when both $W^{2}$ and $Q^{2}$ are large since the vector gluon couplings grow with energy. Part of the final-state phase can be associated with a Wilson line as an augmented LFWF [79] which does not affect the moments.

The Glauber propagation of the vector system $V$ produced by the DDIS interaction on the nuclear front face and its subsequent inelastic interaction with the nucleons in the nuclear interior $V+N_{b} \rightarrow X$ occurs after the lepton interacts with the struck quark. The corresponding amplitude for deeply virtual Compton scattering is not given by the handbag diagram alone since interactions between the two currents are essential. Because of the rescattering dynamics, the DDIS amplitude acquires a complex phase from Pomeron and Regge exchange; thus final-state rescattering corrections lead to nontrivial "dynamical" contributions to the measured PDFs; that is, they are a consequence of the scattering process itself [80]. The $I=1$ Reggeon contribution to DDIS on the front-face nucleon then leads to flavor-dependent antishadowing $[81,82]$. This could explain why the $\mathrm{NuTeV}$ charged current measurement $\mu A \rightarrow \nu X$ scattering does not appear to show antishadowing, in contrast to deep inelastic electron-nucleus scattering as discussed in [83].

Diffractive deep inelastic scattering is leading-twist, and it is an essential component of the two-step amplitude which causes shadowing and antishadowing of the nuclear PDF. It is important to analyze whether the momentum and other sum rules derived from the OPE expansion in terms of local operators remain valid when these dynamical rescattering corrections to the nuclear PDF are included. The OPE is derived assuming that the LF time separation between the virtual photons in the forward virtual Compton amplitude $\gamma^{*} A \rightarrow$ $\gamma^{*} A$ scales as $1 / Q^{2}$. However, the propagation of the vector system $V$ produced by the DDIS interaction on the front face and its inelastic interaction with the nucleons in the nuclear interior $V+N_{b} \rightarrow X$ are characterized by a nonvanishing LF time interval in the nuclear rest frame. Note also that shadowing in deep inelastic lepton scattering on a nucleus involves nucleons facing the incoming lepton beam. The geometrical orientation of the shadowed nucleons is not a property of the nuclear LFWFs used to evaluate the matrix elements of local currents. Thus leading-twist shadowing and antishadowing appear to invalidate the sum rules for nuclear PDFs. The same complications occur in the leading-twist analysis of deeply virtual Compton scattering $\gamma^{*} A \rightarrow \gamma^{*} A$ on a nuclear target. Thus the leading-twist multinucleon processes which produce shadowing and antishadowing in a nucleus are not accounted for using the $Q^{2} \rightarrow \infty$ OPE analysis.

\section{Summary}

The light-front Hamiltonian equation $H_{\mathrm{LF}}|\Psi\rangle=M^{2}|\Psi\rangle$ derived from quantization at fixed LF time $\tau=t+z / c$ provides a causal, Poincaré-invariant method for solving QCD. The eigenvalues $M_{H}^{2}$ are the squares of the hadronic masses, and the eigensolutions provide the LF Fock state wavefunctions $\Psi_{n}\left(x_{i}, \vec{k}_{\perp i}, \lambda_{i}\right)$ controlling hadron dynamics. The LFWFs $\Psi_{n}$ are independent of the hadron's momentum; that is, they are boost invariant and satisfy momentum and spin sum rules. Light-Front Quantization thus provides a physical, frameindependent formalism for hadron dynamics and structure. Observables such as structure functions, transverse momentum distributions, and distribution amplitudes are defined from the hadronic light-front wavefunctions.

The full QCD LF equation can be reduced for massless quarks to an effective LF Shrödinger radial equation for the valence $|q \bar{q}\rangle$ Fock state of $q \bar{q}$ mesons

$$
\left[-\frac{d^{2}}{d \zeta^{2}}+\frac{4 L^{2}-1}{4 \zeta^{2}}+U\left(\zeta^{2}\right)\right] \psi=M^{2} \psi
$$

and similar bound-state equations for baryons, represented as quark + diquark-cluster $|q[q q]\rangle$ eigenstates. The "radial" LF variable $\zeta^{2}=b_{\perp}^{2} x(1-x)$ of LF theory is conjugate to the LF kinetic energy. The identical equation is derived from $\mathrm{AdS}_{5}$, where the fifth coordinate $z$ is identified with $\zeta$ (Light-Front Holography).

The color-confining potential $U\left(\zeta^{2}\right)=\kappa^{4} \zeta^{2}+2 \kappa^{2}(J-1)$ can be derived from soft-wall $\mathrm{AdS}_{5}$ by incorporating the remarkable dAFF principle that a mass scale can appear in the Hamiltonian while retaining the conformal invariance of the action. The result is a color-confining LF potential which depends on a single universal constant $\kappa$ with mass dimensions. In addition, by utilizing superconformal algebra [3], the resulting hadronic color-singlet eigenstates have a $2 \times 2$ representation of mass degenerate bosons and fermions: a $|q \bar{q}\rangle$ meson with $L_{M}=L_{B}+1$, a baryon doublet $|q[q q]\rangle$ with $L_{B}$ and $L_{B}+1$ components of equal weight, and a tetraquark $|[q q][\bar{q} \bar{q}]\rangle$ with $L_{T}=L_{B}$. See Figure 4 . Thus ratios of hadron masses such as $m_{\rho}=M_{p} / \sqrt{2}$ are predicted. The individual contributions LF kinetic energy, potential energy, spin-interactions, and the quark mass to the mass squared of each hadron are also shown. The virial theorem for harmonic oscillator confinement predicts the equality of the LF kinetic and potential contributions to $M_{H}^{2}$ for each hadron.

One obtains new insights into the hadronic spectrum, light-front wavefunctions, and the $e^{-Q^{2} / 4 \kappa^{2}}$ Gaussian functional form of the QCD running coupling in the nonperturbative domain using light-front holography-the duality between the front form and $\mathrm{AdS}_{5}$, the space of isometries 
of the conformal group. AdS/QCD also predicts the analytic form of the nonperturbative running coupling $\alpha_{s}\left(Q^{2}\right) \propto$ $e^{-Q^{2} / 4 \kappa^{2}}$, in agreement with the effective charge measured from measurements of the Bjorken sum rule. This analysis also provides a connection between nonperturbative QCD and PQCD at a scale $Q_{0}$ and a prediction for $\Lambda_{\overline{\mathrm{MS}}}$ from the proton or $\rho$ mass.

Other LF Holographic predictions include the following:

(1) Universal Regge slopes in $n$ and $L$ for mesons: $M^{2}(n, L)=4 \kappa^{2}(n+L)$ for mesons and $M^{2}(n, L)=$ $4 \kappa^{2}(n+L+1)$ for baryons, consistent with measurements.

(2) The pion eigenstate is a massless $q \bar{q}$ bound state for chiral QCD $\left(m_{q}=0\right)$.

(3) Empirically viable predictions for spacelike and timelike hadronic form factors, structure functions, distribution amplitudes, and transverse momentum distributions [84].

(4) Superconformal extensions to heavy-light quark mesons and baryons.

In addition, superconformal algebra leads to remarkable supersymmetric relations between mesons and baryons of the same parity. The mass scale $\kappa$ underlying confinement and hadron masses can be connected to the parameter $\Lambda_{\overline{\mathrm{MS}}}$ in the $\mathrm{QCD}$ running coupling by matching the nonperturbative dynamics, as described by the effective conformal theory mapped to the light-front and its embedding in AdS space, to the perturbative QCD regime. The result is an effective coupling defined at all momenta. This matching of the high and low momentum transfer regimes determines a scale $Q_{0}$ which sets the interface between perturbative and nonperturbative hadron dynamics. The use of $Q_{0}$ to resolve the factorization scale uncertainty for structure functions and distribution amplitudes, in combination with the principle of maximal conformality (PMC) for setting the renormalization scales [70], can greatly improve the precision of perturbative QCD predictions for collider phenomenology. The absence of vacuum excitations of the causal, frame-independent front-form vacuum has important consequences for the cosmological constant. I have also discussed evidence that the antishadowing of nuclear structure functions is nonuniversal, that is, flavor dependent, and why shadowing and antishadowing phenomena may be incompatible with sum rules for nuclear parton distribution functions.

Future work will include the extension of superconformal representations to pentaquark and other exotic hadrons, comparisons with lattice gauge theory predictions, the construction of an AdS/QCD orthonormal basis to diagonalize the QCD light-front Hamiltonian, hadronization at the amplitude level, and the computation of intrinsic heavy quark higher Fock states.

\section{Conflicts of Interest}

The author declares that they have no conflicts of interest.

\section{Acknowledgments}

The author thanks Ralf Hofmann for organizing an outstanding 5th Winter Workshop on Nonperturbative Quantum Field Theory at the Université de Nice. The results presented here are based on collaborations and discussions with Kelly Chiu, Alexandre Deur, Guy de Téramond, Guenter Dosch, Susan Gardner, Fred Goldhaber, Paul Hoyer, Dae Sung Hwang, Rich Lebed, Simonetta Liuti, Cedric Lorcé, Valery Lyubovitskij, Matin Mojaza, Michael Peskin, Craig Roberts, Robert Shrock, Ivan Schmidt, Peter Tandy, and Xing-Gang $\mathrm{Wu}$. This research was supported by the Department of Energy, Contract DE-AC02-76SF00515. SLAC-PUB-17012.

\section{References}

[1] G. F. de Téramond, H. G. Dosch, and S. J. Brodsky, "Baryon spectrum from superconformal quantum mechanics and its light-front holographic embedding," Physical Review D, vol. 91, no. 4, article 045040, 2015.

[2] H. G. Dosch, G. F. de Téramond, and S. J. Brodsky, "Superconformal baryon-meson symmetry and light-front holographic QCD," Physical Review D: Particles, Fields, Gravitation and Cosmology, vol. 91, no. 8, article 085016, 2015.

[3] H. G. Dosch, G. F. de Téramond, and S. J. Brodsky, "Supersymmetry across the light and heavy-light hadronic spectrum," Physical Review D: Particles, Fields, Gravitation and Cosmology, vol. 92, no. 7, article 074010, 2015.

[4] G. F. de Téramond, H. G. Dosch, and S. J. Brodsky, "Kinematical and dynamical aspects of higher-spin bound-state equations in holographic QCD," Physical Review D: Particles, Fields, Gravitation and Cosmology, vol. 87, no. 7, article 075005, 2013.

[5] S. J. Brodsky and R. F. Lebed, "Production of the smallest QED atom: True Muonium $\left(\mu^{+} \mu^{-}\right)$," Physical Review Letters, vol. 102, article 213401, 2009.

[6] A. Banburski and P. Schuster, "Production and discovery of true muonium in fixed-target experiments," Physical Review D: Particles, Fields, Gravitation and Cosmology, vol. 86, article 093007, 2012.

[7] S. J. Brodsky, G. F. de Téramond, A. Deur, and H. G. Dosch, "The light-front schrödinger equation and determination of the perturbative QCD scale from color confinement," Few-Body Systems, vol. 56, no. 6-9, article 621, 2015.

[8] V. de Alfaro, S. Fubini, and G. Furlan, "Conformal invariance in quantum mechanics," Il Nuovo Cimento A, vol. 34, no. 4, pp. 569-612, 1976.

[9] S. J. Brodsky, G. F. de Téramond, and H. G. Dosch, "Threefold complementary approach to holographic QCD," Physics Letters $B$, vol. 729, pp. 3-8, 2014.

[10] S. J. Brodsky, "Advances in light-front QCD: supersymmetric properties of hadron physics from light-front holography and superconformal algebra," Few-Body Systems, vol. 58, no. 133, 2017.

[11] S. J. Brodsky, "Light-front holography, color confinement, and supersymmetric features of QCD," Few-Body Systems, vol. 57, no. 8,2016 .

[12] S. J. Brodsky, "New insights into color confinement, hadron dynamics, spectroscopy, and jet hadronization from lightfront holography and superconformal algebra," Russian Physics Journal, vol. 60, no. 3, pp. 399-416, 2017. 
[13] P. A. Dirac, "Forms of relativistic dynamics," Reviews of Modern Physics, vol. 21, pp. 392-399, 1949.

[14] S. J. Brodsky, H.-C. Pauli, and S. S. Pinsky, "Quantum chromodynamics and other field theories on the light cone," Physics Reports, vol. 301, no. 4-6, pp. 299-486, 1998.

[15] H. Reinhardt, "Wilson loop in light-front quantization," Physical Review D, vol. 95, no. 4, article 045015, 2017.

[16] J. Terrell, "Invisibility of the Lorentz contraction," Physical Review A: Atomic, Molecular and Optical Physics, vol. 116, no. 4, pp. 1041-1045, 1959.

[17] R. Penrose, "The apparent shape of a relativistically moving sphere," Proceedings of the Cambridge Philosophical Society, vol. 55, pp. 137-139, 1959.

[18] R. Haag, J. T. Lopuszanski, and M. Sohnius, "All possible generators of supersymmetries of the S-matrix," Nuclear Physics $B$, vol. 88, pp. 257-274, 1975.

[19] S. Fubini and E. Rabinovici, "Superconformal quantum mechanics," Nuclear Physics B, vol. 245, no. C, pp. 17-44, 1984.

[20] G. 't Hooft, "Minimal Strings for Baryons," https://arxiv.org/abs/ hep-th/0408148.

[21] T. Liu and B. Q. Ma, "Baryon properties from light-front holographic QCD," Physical Review D, vol. 92, no. 9, article 096003, 2015.

[22] H. G. Dosch, G. F. de Téramond, and S. J. Brodsky, "Supersymmetry across the light and heavy-light hadronic spectrum II," Physical Review D, vol. 95, no. 3, article 034016, 2017.

[23] S. J. Brodsky, M. Diehl, and D. S. Hwang, "Light-cone wavefunction representation of deeply virtual compton scattering," Nuclear Physics B, vol. 596, no. 1-2, pp. 99-124, 2001.

[24] S. J. Brodsky, D. S. Hwang, B. Q. Ma, and I. Schmidt, "Lightcone representation of the spin and orbital angular momentum of relativistic composite systems," Nuclear Physics B, vol. 593, pp. 311-335, 2001.

[25] I. Y. Kobzarev and L. B. Okun, "Gravitational interaction of fermions," Soviet Physics-Journal of Experimental and Theoretical Physics, vol. 16, pp. 1343-1346, 1963.

[26] O. V. Teryaev, "Spin structure of nucleon and equivalence principle," https://arxiv.org/abs/hep-ph/9904376.

[27] S. J. Brodsky, F. Cao, and G. F. de Téramond, "Evolved QCD predictions for the meson-photon transition form factors," Physical Review D: Particles, Fields, Gravitation and Cosmology, vol. 84, no. 3, article 075012, 2011.

[28] J. R. Forshaw and R. Sandapen, "AdS/QCD holographic wave function for the $\rho$ Meson and diffractive $\rho$ Meson electroproduction," Physical Review Letters, vol. 109, no. 8, article 081601, 2012.

[29] S. J. Brodsky, G. F. de Téramond, H. G. Dosch, and J. Erlich, "Light-front holographic QCD and emerging confinement," Physics Reports, vol. 584, pp. 1-105, 2015.

[30] T. Gutsche, V. E. Lyubovitskij, I. Schmidt, and A. Vega, "Nuclear physics in soft-wall AdS/QCD: deuteron electromagnetic form factors," Physical Review D, vol. 91, no. 11, article 114001, 2015.

[31] T. Gutsche, V. E. Lyubovitskij, and I. Schmidt, "Deuteron electromagnetic structure functions and polarization properties in soft-wall AdS/QCD," Physical Review D, vol. 94, no. 11, article 116006, 2016.

[32] G. F. de Téramond and S. J. Brodsky, "Light-front holography: a first approximation to QCD," Physical Review Letters, vol. 102, no. 8 , article $081601,2009$.
[33] A. V. Smirnov, V. A. Smirnov, and M. Steinhauser, "Three-loop static potential," Physical Review Letters, vol. 104, no. 11, article $112002,2010$.

[34] D. Ashery, "Measurement of light-cone wave functions by diffractive dissociation," Nuclear Physics B-Proceedings Supplements, vol. 108, pp. 321-326, 2002.

[35] P. P. Srivastava and S. J. Brodsky, "Light-front formulation of the standard model," Physical Review D: Particles, Fields, Gravitation and Cosmology, vol. 66, no. 4, article 045019, 2002.

[36] V. N. Gribov and L. N. Lipatov, "Deep inelastic ep scattering in perturbation theory," Soviet Journal of Nuclear Physics, vol. 15, no. 4, pp. 438-450, 1972.

[37] G. Altarelli and G. Parisi, "Asymptotic freedom in parton language," Nuclear Physics B, vol. 126, no. 2, pp. 298-318, 1977.

[38] Y. L. Dokshitzer, "Calculation of the structure functions for deep inelastic scattering and $\mathrm{e}^{+} \mathrm{e}^{-}$annihilation by perturbation theory in quantum chromodynamics," Journal of Experimental and Theoretical Physics, vol. 73, pp. 1216-1240, 1977.

[39] G. P. Lepage and S. J. Brodsky, "Exclusive processes in quantum chromodynamics: evolution equations for hadronic wave functions and the form-factors of mesons," Physics Letters, vol. 87B, no. $359,1979$.

[40] G. P. Lepage and S. J. Brodsky, "Exclusive processes in perturbative quantum chromodynamics," Physical Review D: Particles, Fields, Gravitation and Cosmology, vol. 22, article 2157, 1980.

[41] A. V. Efremov and A. V. Radyushkin, "Factorization and asymptotical behavior of pion form-factor in QCD," Physics Letters, vol. 94B, no. 245, 1980.

[42] A. V. Efremov and A. V. Radyushkin, "Asymptotical behavior of pion electromagnetic form-factor in QCD," Theoretical and Mathematical Physics, vol. 42, no. 147, 1980.

[43] S. J. Brodsky and S. Gardner, “Comment on 'New limits on intrinsic charm in the nucleon from global analysis of parton distributions"' Physical Review Letters, vol. 116, no. 1, article 019101, 2016.

[44] H.-C. Pauli and S. J. Brodsky, "Solving field theory in one space and one time dimension," Physical Review D: Particles, Fields, Gravitation and Cosmology, vol. 32, no. 8, pp. 1993-2000, 1985.

[45] K. Hornbostel, S. J. Brodsky, and H.-C. Pauli, "Light-conequantized QCD in $1+1$ dimensions," Physical Review D: Particles, Fields, Gravitation and Cosmology, vol. 41, no. 12, pp. 3814-3821, 1990.

[46] J. P. Vary, X. Zhao, A. Ilderton, H. Honkanen, P. Maris, and S. J. Brodsky, "Applications of basis light-front quantization to QED," Nuclear Physics B-Proceedings Supplements, vol. 251252, no. 10, 2014.

[47] S. J. Brodsky, A. L. Deshpande, H. Gao, R. D. McKeown, C. A. Meyer et al., "QCD and hadron physics," https://arxiv.org/abs/ 1502.05728.

[48] J. P. Vary, X. Zhao, A. Ilderton, H. Honkanen, P. Maris, and S. J. Brodsky, "Basis light-front quantization: a new approach to non-perturbative scattering and time-dependent production processes," Acta Physica Polonica B Proceedings Supplement, vol. 6, no. 257, 2013.

[49] D. Ashery, "Measurement of the photon light-cone wave function by diffractive dissociation," Nuclear Physics B-Proceedings Supplements, vol. 161, pp. 8-14, 2006.

[50] S. J. Brodsky, C. Ji, and G. P. Lepage, "Quantum chromodynamic predictions for the deuteron form factor," Physical Review Letters, vol. 51, no. 83, 1983. 
[51] C. Cruz-Santiago, P. Kotko, and A. M. Staśto, "Scattering amplitudes in the light-front formalism," Progress in Particle and Nuclear Physics, vol. 85, pp. 82-131, 2015.

[52] K. Y. J. Chiu and S. J. Brodsky, "Angular momentum conservation law in light-front quantum field theory," Physical Review D, vol. 95, no. 6, article 065035, 2017.

[53] S. J. Brodsky, R. Roskies, and R. Suaya, "Quantum electrodynamics and renormalization theory in the infinite-momentum frame," Physical Review D: Particles, Fields, Gravitation and Cosmology, vol. 8, no. 12, pp. 4574-4594, 1973.

[54] S. J. Brodsky and G. F. de Téramond, "Light-front holography and QCD hadronization at the amplitude level," https://arxiv .org/abs/0901.0770.

[55] R. L. Jaffe, "The axial anomaly and the sum rules for spin dependent electroproduction," Physics Letters B, vol. 193, no. 101, 1987.

[56] S. J. Brodsky, C. D. Roberts, R. Shrock, and P. C. Tandy, "Confinement contains condensates," Physical Review C: Nuclear Physics, vol. 85, article 065202, 2012.

[57] A. Zee, "Dark energy and the cosmological constant paradox," Modern Physics Letters A, vol. 23, no. 1336, 2008.

[58] H. Reinhardt and H. Weigel, "Vacuum nature of the QCD condensates," Physical Review D, vol. 85, no. 7, article 074029, 2012.

[59] A. Casher and L. Susskind, "Chiral magnetism (or magnetohadrochironics)," Physical Review D: Particles, Fields, Gravitation and Cosmology, vol. 9, article 436, 1974.

[60] S. J. Brodsky and R. Shrock, "Condensates in quantum chromodynamics and the cosmological constant," Proceedings of the National Academy of Sciences, vol. 108, pp. 45-50, 2011.

[61] S. J. Brodsky, C. D. Roberts, R. Shrock, and P. C. Tandy, "New perspectives on the quark condensate," Physical Review C: Nuclear Physics, vol. 82, article 022201, 2010.

[62] E. P. Verlinde, "Emergent gravity and the dark universe," 2017, https://arxiv.org/abs/1611.02269.

[63] G. Grunberg, "Renormalization scheme independent QCD and QED: the method of effective charges," Physical Review D, vol. 29, no. 10, pp. 2315-2338, 1984.

[64] S. J. Brodsky and H. J. Lu, "Commensurate scale relations in quantum chromodynamics," Physical Review D: Particles, Fields, Gravitation and Cosmology, vol. 51, pp. 3652-3668, 1995.

[65] S. J. Brodsky, G. F. de Téramond, and A. Deur, "Nonperturbative QCD coupling and its $\beta$ function from light-front holography," Physical Review D: Particles, Fields, Gravitation and Cosmology, vol. 81, article 096010, 2010.

[66] A. Deur, V. Burkert, J. Chen, and W. Korsch, "Experimental determination of the effective strong coupling constant," Physics Letters B, vol. 650, pp. 244-248, 2007.

[67] A. Deur, S. J. Brodsky, and G. F. de Teramond, "Connecting the hadron mass scale to the fundamental mass scale of quantum chromodynamics," Physics Letters B, vol. 750, pp. 528-532, 2015.

[68] K. A. Olive, K. Agashe, C. Amsler, and Particle Data Group, "Review of particle physics," Chinese Physics C, vol. 38, no. 9, Article ID 090001, 2014.

[69] A. Zee, "Quantum field theory in a nutshell," Princeton, UK: Princeton University Pr. 576 p, 2010.

[70] M. Mojaza, S. J. Brodsky, and X. Wu, "Systematic all-orders method to eliminate renormalization-scale and scheme ambiguities in Perturbative QCD," Physical Review Letters, vol. 110, article 192001, 2013.
[71] S. J. Brodsky and S. D. Drell, "Anomalous magnetic moment and limits on fermion substructure," Physical Review D: Particles, Fields, Gravitation and Cosmology, vol. 22, no. 9, article 2236, 1980.

[72] S. Liuti, A. Rajan, A. Courtoy, G. R. Goldstein, and J. O. G. Hernandez, "Partonic picture of generalized transverse momentum distributions," International Journal of Modern Physics: Conference Series, vol. 25, article 1460009, 2014.

[73] C. Mondal and D. Chakrabarti, "Generalized parton distributions and transverse densities in a light-front quark-diquark model for the nucleons," The European Physical Journal C, vol. 75, no. 6, article 261, 2015.

[74] C. Lorcé, B. Pasquini, and M. Vanderhaeghen, "Unified framework for generalized and transverse-momentum dependent parton distributions within a $3 \mathrm{Q}$ light-cone picture of the nucleon," Journal of High Energy Physics, vol. 1105, no. 041, 2011.

[75] S. J. Brodsky, "Dynamic versus static structure functions and novel diffractive effects in QCD," AIP Conference Proceedings, vol. 1105, no. 315, 2009.

[76] S. J. Brodsky, "Dynamic versus static hadronic structure functions," Nuclear Physics A, vol. 827, no. 327C, 2009.

[77] S. J. Brodsky, D. S. Hwang, and I. Schmidt, "Final-state interactions and single-spin asymmetries in semi-inclusive deep inelastic scattering," Physics Letters B, vol. 530, pp. 99-107, 2002.

[78] S. J. Brodsky, P. Hoyer, N. Marchal, S. Peigné, and F. Sannino, "Structure functions are not parton probabilities," Physical Review D: Particles, Fields, Gravitation and Cosmology, vol. 65, article 114025, 2002.

[79] S. J. Brodsky, B. Pasquini, B. Xiao, and F. Yuan, "Phases of augmented hadronic light-front wave functions," Physics Letters $B$, vol. 687, pp. 327-330, 2010.

[80] S. J. Brodsky, D. S. Hwang, Y. V. Kovchegov, I. Schmidt, and M. D. Sievert, "Single-spin asymmetries in semi-inclusive deep inelastic scattering and Drell-Yan processes," Physical Review D: Particles, Fields, Gravitation and Cosmology, vol. 88, no. 1, article 014032, 2013.

[81] S. J. Brodsky and H. J. Lu, "Shadowing and antishadowing of nuclear structure functions," Physical Review Letters, vol. 64, article 1342, 1990.

[82] S. J. Brodsky, I. Schmidt, and J. Yang, "Nuclear antishadowing in neutrino deep inelastic scattering," Physical Review D: Particles, Fields, Gravitation and Cosmology, vol. 70, article 116003, 2004.

[83] I. Schienbein, J. Y. Yu, C. Keppel, J. G. Morfín, F. Olness, and J. F. Owens, "Nuclear parton distribution functions from neutrino deep inelastic scattering," Physical Review D: Particles, Fields, Gravitation and Cosmology, vol. 77, article 054013, 2008.

[84] R. S. Sufian, G. F. de Téramond, S. J. Brodsky, A. Deur, and H. G. Dosch, "Analysis of nucleon electromagnetic form factors from light-front holographic QCD: The spacelike region," Physical Review D: Particles, Fields, Gravitation and Cosmology, vol. 95, no. 1, article 014011, 2017. 

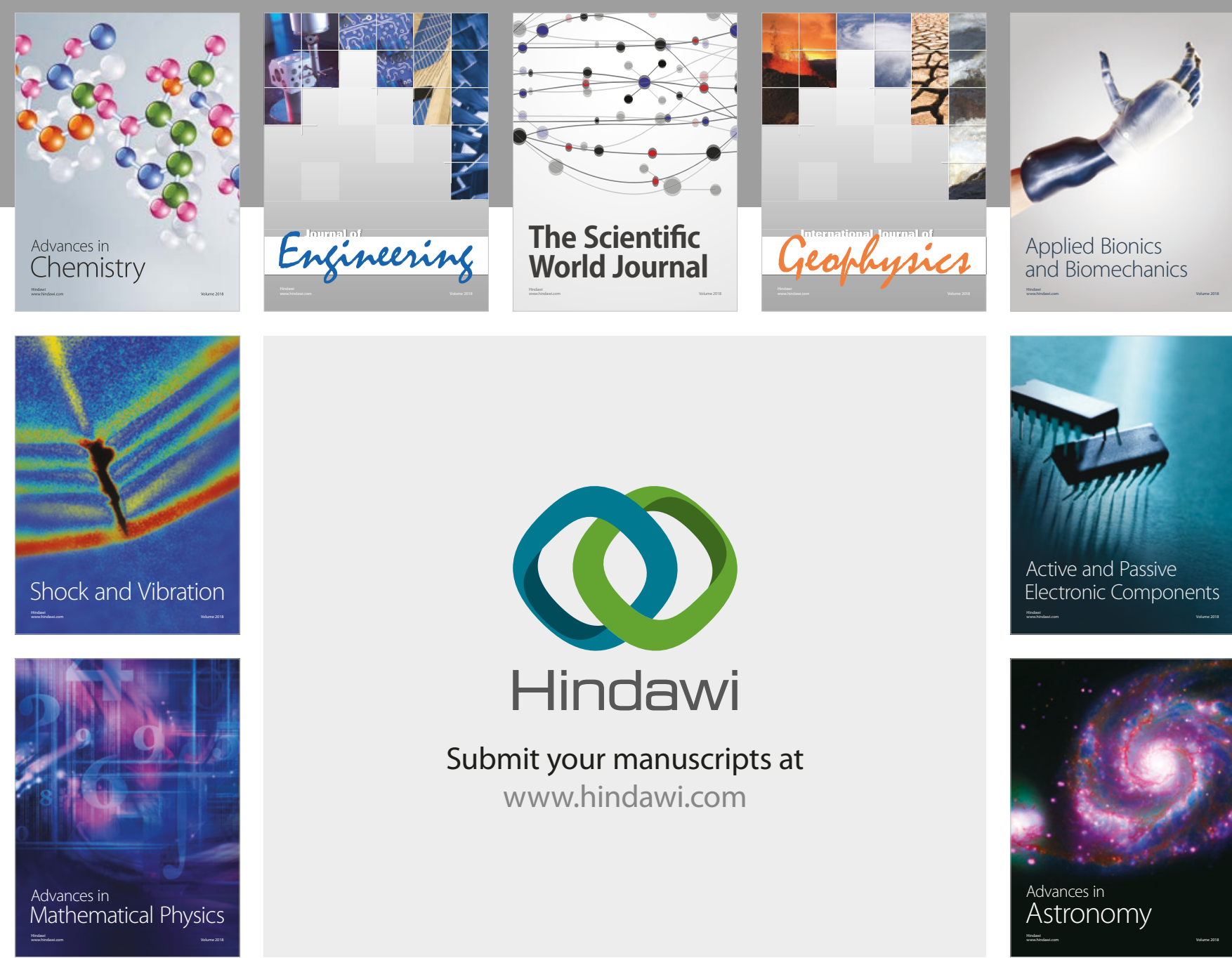

Submit your manuscripts at

www.hindawi.com

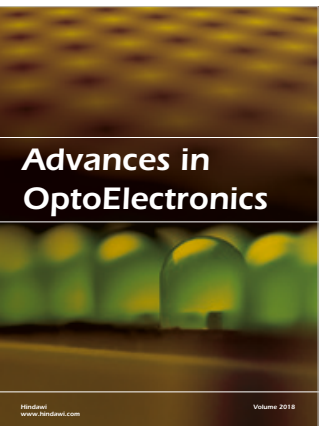

\section{Rotcting Machinery}
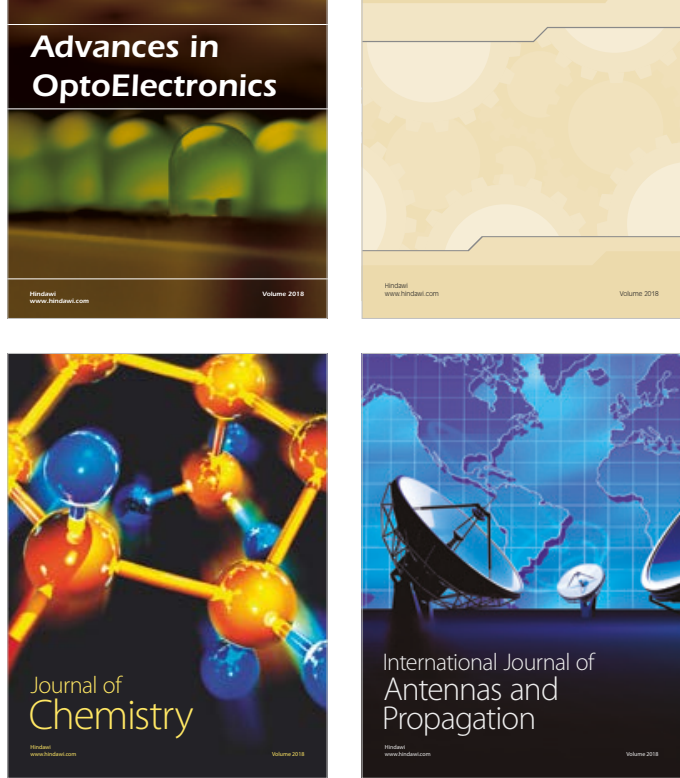

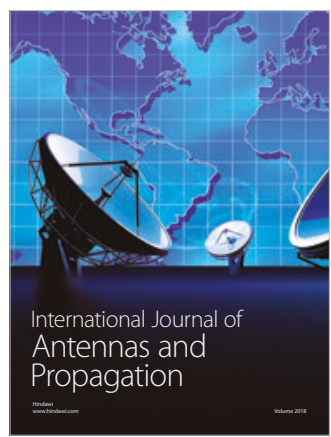

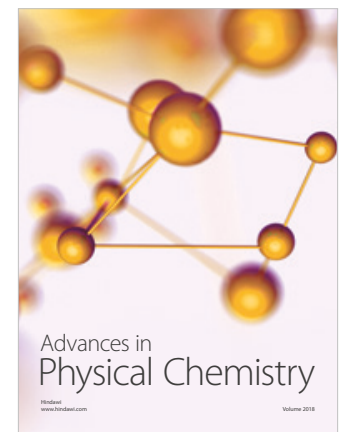

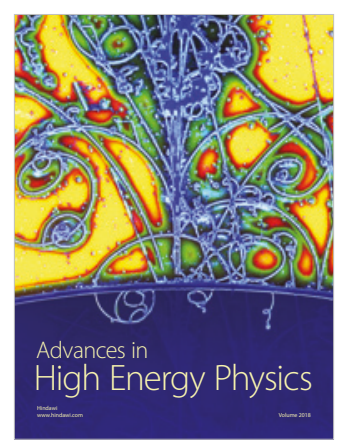

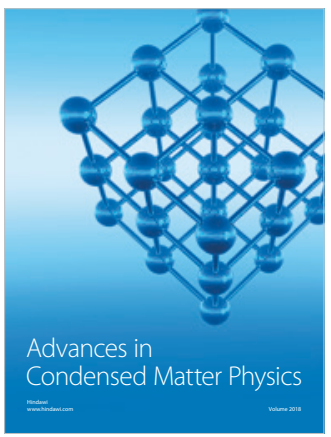

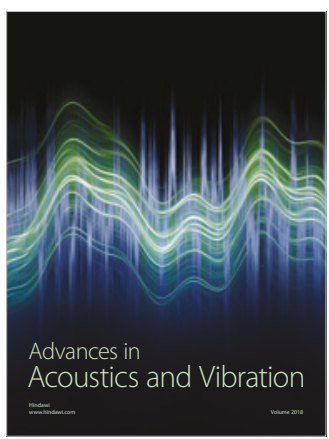

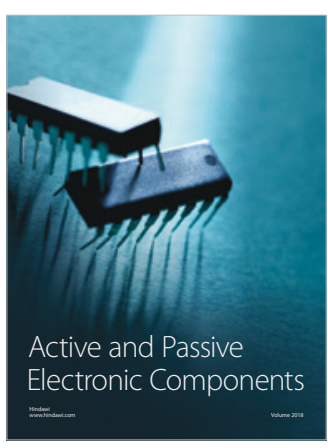
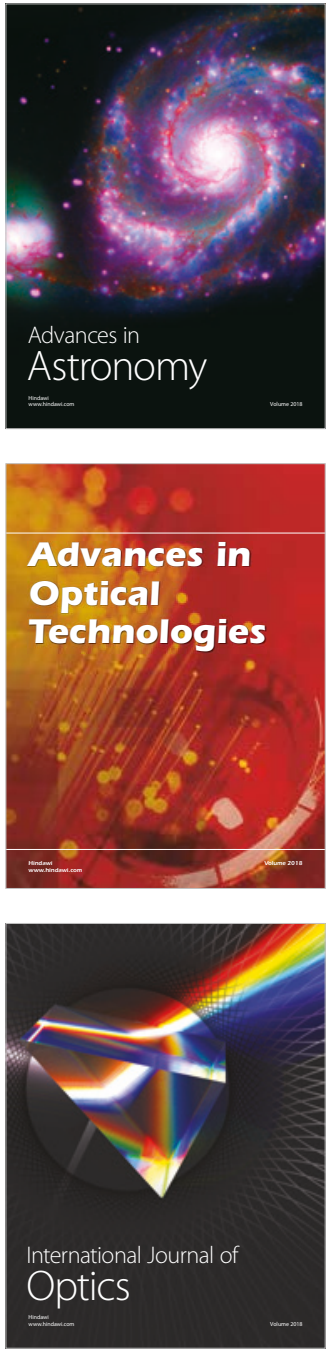